\title{
Recent developments in the theory of Borel reducibility
}

\author{
by \\ Greg Hjorth (Los Angeles, CA) and \\ Alexander S. Kechris (Pasadena, CA)
}

\begin{abstract}
Let $E_{0}$ be the Vitali equivalence relation and $E_{3}$ the product of countably many copies of $E_{0}$. Two new dichotomy theorems for Borel equivalence relations are proved. First, for any Borel equivalence relation $E$ that is (Borel) reducible to $E_{3}$, either $E$ is reducible to $E_{0}$ or else $E_{3}$ is reducible to $E$. Second, if $E$ is a Borel equivalence relation induced by a Borel action of a closed subgroup of the infinite symmetric group that admits an invariant metric, then either $E$ is reducible to a countable Borel equivalence relation or else $E_{3}$ is reducible to $E$.

We also survey a number of results and conjectures concerning the global structure of reducibility on Borel equivalence relations.
\end{abstract}

1. Introduction. In this paper we present the proofs of the results announced in [12] and survey the recent work bearing on the sweeping conjectures which were presented in that paper.

2. Definitions. We briefly recall the relevant definitions. This is only a skeleton of the introduction of [12], which also presents considerable motivation.

Definition 2.1. A topological space is said to be Polish if it is separable and the topology is generated by some complete metric. The Borel subsets of a Polish space are those contained in the $\sigma$-algebra generated by the open sets.

An equivalence relation $E \subseteq X \times X$ on a Polish space $X$ is said to be Borel if it appears in the $\sigma$-algebra generated by the open sets in the product topology on $X \times X$. Here we have not really departed from our original use of the term "Borel", since $X \times X$ is a Polish space in this product topology.

2000 Mathematics Subject Classification: Primary 03E15.

Research of the first author partially supported by NSF Grant DMS 96-22977.

Research of the second author partially supported by NSF Grant DMS 96-19880. 
A function $f: X \rightarrow Y$ between Polish spaces is said to be Borel if the preimage of any open set is Borel. It follows from classical techniques (see [14], §18.C) that this is equivalent to requiring that the graph of $f$ be Borel as a subset of $X \times Y$.

Definition 2.2. For $E$ and $F$ Borel equivalence relations on Polish spaces $X$ and $Y$, we say that $E$ is Borel reducible to $F$, written

$$
E \leq_{\mathrm{B}} F \text {, }
$$

if there is a Borel function $f: X \rightarrow Y$ such that for all $x_{1}, x_{2} \in X$,

$$
x_{1} E x_{2} \Leftrightarrow f\left(x_{1}\right) F f\left(x_{2}\right) .
$$

This definition naturally gives rise to variations. We write $E \not_{\mathrm{B}} F$ if it is not the case that $E \leq_{\mathrm{B}} F$. We write

$$
E<_{\mathrm{B}} F
$$

if we have both $E \leq_{\mathrm{B}} F$ and $F \underline{B}_{\mathrm{B}} E$. We write

$$
E \sim_{\mathrm{B}} F
$$

if there is a reduction in both directions:

$$
E \leq_{\mathrm{B}} F, \quad F \leq_{\mathrm{B}} E .
$$

We say that $E$ and $F$ are Borel incomparable if there is reduction in neither direction:

$$
E \not \mathbb{B}_{\mathrm{B}} F, \quad F \not \mathbb{B}_{\mathrm{B}} E \text {. }
$$

For $E$ an equivalence relation on a space $X$ and $x \in X$, we let $[x]_{E}=$ $\{y \in X: x E y\}$ denote the equivalence class of $x$. We can then let $X / E=$ $\left\{[x]_{E}: x \in X\right\}$ indicate the collection of all equivalence classes.

The first comment that must be made about that partial order (Borel equivalence relations, $\leq_{\mathrm{B}}$ ) is that it is massively complicated and apparently resistant to any global structure theorems. For instance:

TheOREM 2.3 (Louveau-Veličković; see [18]). There is an assignment $S \mapsto E_{S}$ of Borel equivalence relations to subsets of $\mathbb{N}$ such that for all $S, T \subseteq \mathbb{N}, E_{S} \leq E_{T}$ if and only if $S \backslash T$ is finite.

Definition 2.4. For $X$ a Polish space, we let $\Delta(X)$ denote the equivalence relation of equality on $X$ :

$$
\Delta(X)=\left\{\left(x_{1}, x_{2}\right) \in X^{2}: x_{1}=x_{2}\right\} .
$$

Since $X / \Delta(X)$ screams out to be identified with $X$, we will frequently slur over the distinction between $X$ and $\Delta(X)$. In particular, we will use $n$ to denote

$$
\Delta(\{0,1, \ldots, n-1\})
$$


the equality relation on the discrete space $\{0,1, \ldots, n-1\}$ of size $n$, and $\mathbb{N}$ to denote equality on the countably infinite discrete space $\mathbb{N}=\{0,1, \ldots\}$, and $\mathbb{R}$ to denote equality on the set of reals. Note that any countable discrete space is Polish, and thus

$$
0,1, \ldots, n, \ldots, \mathbb{N}, \mathbb{R}
$$

can be thought of as the simplest examples of Borel equivalence relations on Polish spaces. It was shown in [19] that for every Borel equivalence relation $E$, either $E$ is $\sim_{\mathrm{B}}$ to one of $1,2, \ldots, n, \ldots, \mathbb{N}$ or else $\mathbb{R} \leq_{\mathrm{B}} E$.

Slightly more complicated is the equivalence relation of eventual agreement on infinite binary sequences. So for

$$
x, y \in 2^{\mathbb{N}}:=\{z \mid z: \mathbb{N} \rightarrow\{0,1\}\},
$$

we set

$$
x E_{0} y \quad \text { iff } \quad \exists k \forall n>k(x(n)=y(n)) .
$$

We may view $2^{\mathbb{N}}$ as a Polish space by taking the discrete topology on $2:=$ $\{0,1\}$ and the resulting product topology on $2^{\mathbb{N}}$. It was shown in [8] that $E_{0}$ is the next Borel equivalence relation after $\mathbb{R}$.

After $E_{0}$ the ordering fans out. The first Borel equivalence relation here to be seriously studied was the equivalence relation of eventual agreement on sequences of points in the Cantor space $2^{\mathbb{N}}$. For

$$
x, y \in\left(2^{\mathbb{N}}\right)^{\mathbb{N}}:=\left\{z \mid z: \mathbb{N} \rightarrow 2^{\mathbb{N}}\right\},
$$

we set

$$
x E_{1} y \quad \text { iff } \quad \exists k \forall n>k(x(n)=y(n)) .
$$

The paper [16] showed that $E_{0}<_{\mathrm{B}} E_{1}$ and there is no Borel $E$ with $E_{0}<_{\mathrm{B}}$ $E<_{\mathrm{B}} E_{1}$.

Also strictly above $E_{0}$ is the equivalence relation $\left(E_{0}\right)^{\mathbb{N}}$ obtained by taking its countable product. So for $x, y \in\left(2^{\mathbb{N}}\right)^{\mathbb{N}}$ we set

$$
x E_{3} y \text { iff } \forall n\left(x(n) E_{0} y(n)\right) .
$$

It is folklore that $E_{0}<_{\mathrm{B}} E_{3}$ and it follows from [16] that $E_{1}$ and $E_{3}$ are Borel incomparable. We announced in [12] that there is no $E$ with $E_{0}<_{\mathrm{B}}$ $E<_{\mathrm{B}} E_{3}$, and we will give the proof below.

In passing from $E_{1}$ to $E_{3}$ we skipped over $E_{2}$. In fact, its construction is less obvious than these other examples, and so we will postpone giving the usual definiton until we come to the subject of Polishable ideals. In the meantime it might be worth saying that, up to $\sim_{\mathrm{B}}$-equivalence, $E_{2}$ is given by the coset equivalence relation of

$$
\ell^{1}:=\left\{x \in \mathbb{R}^{\mathbb{N}}: \sum|x(i)|<\infty\right\}
$$

thought of as a subgroup of $\mathbb{R}^{\mathbb{N}}$ in the usual way. 
Definition 2.5. A Borel equivalence relation $E$ on $X$ is said to be countable if every equivalence class is countable. It is then said to be treeable if there is a symmetric Borel relation $R \subseteq X \times X$ which has no cycles and whose connected components form the equivalence classes of $E$; in other words, we may, in a Borel manner, place the structure of a tree on each $[x]_{E}$.

An equivalence relation $E$ is said to be smooth or concretely classifiable if it is Borel reducible to $\mathbb{R}$.

It is a non-trivial fact that in the $\leq_{\mathrm{B}}$ ordering there is a maximal countable Borel equivalence relation, $E_{\infty}$, and a maximal countable treeable Borel equivalence relation, $E_{\mathrm{T} \infty}$. For the sake of definiteness, we give each one an instantiation, but the reader should see [4] or [13] for a more detailed analysis.

Definition 2.6. Let $\mathbb{F}_{2}$ denote the free group on two generators and let $2^{\mathbb{F}_{2}}$ denote the space of all functions $f: \mathbb{F}_{2} \rightarrow\{0,1\}$, equipped with the product topology (under which it is isomorphic to the Cantor space $2^{\mathbb{N}}$ ). We let $\mathbb{F}_{2}$ act on $2^{\mathbb{F}_{2}}$ by the shift action

$$
(\sigma \cdot f)(\tau)=f\left(\sigma^{-1} \tau\right)
$$

for $f \in 2^{\mathbb{F}_{2}}$ and $\sigma, \tau \in \mathbb{F}_{2}$. For any two $f_{1}, f_{2}: \mathbb{F}_{2} \rightarrow\{0,1\}$ we set

$f_{1} E_{\infty} f_{2} \quad$ iff there is some $\sigma \in \mathbb{F}_{2}$ with $\sigma \cdot f_{1}=f_{2}$.

We then obtain the universal treeable equivalence relation by restricting to the set on which the action is free. So first let $F\left(2^{\mathbb{F}_{2}}\right)$ be the set of functions $f$ for which, whenever $\sigma \in \mathbb{F}_{2}$ is not the identity,

$$
\sigma \cdot f \neq f \text {. }
$$

This set of points is a $G_{\delta}$ subset of $2^{\mathbb{N}}$ and hence a Polish space (see [14], 3.C). The relation $E_{\mathrm{T} \infty}$ is the restriction of $E_{\infty}$ to the set $F\left(2^{\mathbb{F}_{2}}\right)$.

The notation $E_{\infty}$ is somewhat misleading, since it is $\geq_{\mathrm{B}} E_{0}$ but not $\geq_{\mathrm{B}} E_{1}, E_{2}$, or $E_{3}$.

Definition 2.7. We let $p(\mathbb{N})$ denote the collection of all subsets of the natural numbers. A collection $I \subseteq p(\mathbb{N})$ is said to be an ideal if it is closed under finite unions and the process of passing to a subset of a member of $I$. We can view $p(\mathbb{N})$ as a Polish space in the natural way, by identifying it with $2^{\mathbb{N}}$ via the association of the characteristic function to a subset of $\mathbb{N}$; in other words, we give it the topology generated by taking as basic open sets those of the form

$$
\left\{A \subseteq \mathbb{N}: \forall i \in F_{0}(i \notin A), \forall i \in F_{1}(i \in A)\right\},
$$

where $F_{0}, F_{1}$ are finite sets of natural numbers. It will be convenient to identify $p(\mathbb{N})$ with $2^{\mathbb{N}}$. 
Any ideal can be viewed as an abelian group under the operation of symmetric difference. Thus for $A, B \subseteq \mathbb{N}$ we let

$$
A+B=(A \backslash B) \cup(B \backslash A) .
$$

A Borel ideal $I$ on $p(\mathbb{N})$ is said to be Polishable if there is a Polish topology $\tau$ on $I$ such that

(i) $(I, \tau,+)$ is a Polish group, that is to say, the operation of symmetric difference is continuous with respect to $\tau$;

(ii) $\tau$ gives rise to the original Borel structure on $I$, that is to say, a set $X \subseteq I$ appears in the $\sigma$-algebra generated by the $\tau$-open sets if and only if it is Borel with respect to the above Polish topology on $2^{\mathbb{N}}$.

Sławomir Solecki in [20] has shown that all Polishable ideals are $F_{\sigma \delta}$, and that the $F_{\sigma}$ Polishable ideals are those which may be represented as sets which are finite for some appropriately chosen "exhaustive" lower semicontinuous submeasure on $\mathbb{N}$.

If $I$ is an ideal on $p(\mathbb{N})$, then we let $E_{I}$ denote the corresponding coset equivalence relation on $2^{\mathbb{N}}$; thus

$$
x E_{I} y \quad \text { iff } \quad\{n: x(n) \neq y(n)\} \in I .
$$

Definition 2.8. We let $\mathcal{I}_{(1 / n)}$ denote the summable ideal, where for $A$ $\subseteq \mathbb{N}$ we have $A \in \mathcal{I}_{(1 / n)}$ if

$$
\sum_{n \in A} \frac{1}{n+1}<\infty .
$$

With this in hand we can finally define $E_{2}$ to be equal to $\mathcal{I}_{(1 / n)}$, the coset equivalence relation arising from $\mathcal{I}_{(1 / n)}$ in $2^{\mathbb{N}}$.

Unlike $E_{1}$ and $E_{3}$, we are still only able to conjecture that there is no $E$ strictly $\left(\right.$ in $<_{\mathrm{B}}$ ) between $E_{0}$ and $E_{2}$. However, [9] comes close to proving this.

Definition 2.9. A topological group is said to be Polish if the underlying topological space is Polish. If $G$ is a Polish group equipped with a continuous (resp. Borel) action on a Polish space $X$, then we say $X$ is a Polish (resp. Borel) G-space. We then denote the orbit equivalence relation by $E_{G}^{X}$, so that

$$
x_{1} E_{G}^{X} x_{2} \quad \text { iff } \quad \text { there is some } g \in G \text { with } g \cdot x_{1}=x_{2} .
$$

Many Borel equivalence relations arise in this form, or are at least Borel reducible to the orbit equivalence relation of some Polish group acting Borel on a Polish space. It was shown in [16] that whenever $G$ is a Polish group and $X$ is a Borel $G$-space, $E_{1} \not_{\mathrm{B}} E_{G}^{X}$. Consequently, whenever $E_{1} \leq_{\mathrm{B}} E$ we deduce that $E$ fails to be Borel reducible to any such $E_{G}^{X}$; it remains open 
whether this is the only reason a Borel equivalence relation may fail to be Borel reducible to a Borel Polish group action.

An important class of Polish group actions are those presented by $S_{\infty}$, the group of all permutations of $\mathbb{N}$ equipped with the topology of pointwise convergence. Appropriately understood, the isomorphism relation on countable structures can be viewed as the orbit equivalence relation induced by an action of $S_{\infty}$ (see for instance [10], §2.3). There is a fundamental kind of obstruction to reduction to the orbit equivalence relations of the form $E_{S_{\infty}}^{X}$.

Definition 2.10. A continuous action of a Polish group $G$ on a Polish space $X$ is said to be turbulent if:

(i) every orbit is dense;

(ii) every orbit is meager;

(iii) for all $x, y \in X, U \subseteq X, V \subseteq G$ open with $x \in U, 1 \in V$, there exists $y_{0} \in[y]_{G}:=G \cdot y$ and $\left(g_{i}\right)_{i \in \mathbb{N}} \subseteq V,\left(x_{i}\right)_{i \in \mathbb{N}} \subseteq U$ with

$$
x_{0}=x, \quad x_{i+1}=g_{i} \cdot x_{i},
$$

and for some subsequence $\left(x_{n(i)}\right)_{i \in \mathbb{N}}$,

$$
x_{n(i)} \rightarrow y_{0}
$$

In [10] it is shown that an orbit equivalence relation arising from a turbulent action of a Polish group is never reducible to the orbit equivalence relation $E_{S_{\infty}}^{X}$ arising from a Borel action of the infinite symmetric group on some Polish space $X$.

3. The countable equivalence relations. The paper [12] bemoaned the failure to find two $\leq_{\mathrm{B}}$-incomparable countable Borel equivalence relations. At the end of 1998 this was finally settled by Scott Adams and Alexander Kechris, who used the superrigidity theory of Zimmer [23], in the ergodic theory of higher-rank linear algebraic groups, to show that such examples exist and they exist in abundance. For instance, their methods were easily sufficient to obtain a Louveau-Veličković type result:

THEOREM 3.1 (Adams-Kechris; see [1]). There is an assignment $S \mapsto$ $E_{S}$ of countable Borel equivalence relations to subsets of $\mathbb{N}$ such that for all $S, T \subseteq \mathbb{N}$ we have $E_{S} \leq E_{T}$ if and only if $S \backslash T$ is finite.

The examples obtained by their methods are all non-treeable. Thus the original problem lives on in a weaker form:

Question 3.2. Do there exist $\leq_{\mathrm{B}}$-incomparable treeable countable Borel equivalence relations?

4. The summable ideal. Independently, Ilijas Farah and Boban Veličković refuted Conjecture 3 from [12] regarding the equivalence relation induced by the cosets of the summable ideal in $p(\mathbb{N})$ : 
Theorem 4.1 (Farah, Veličković; see [5], [22]). There is an $F_{\sigma}$ Polishable ideal $\mathcal{I}_{\text {Tsir }} \subseteq p(\mathbb{N})$ such that

$$
E_{0}<_{\mathrm{B}} E_{\mathcal{I}_{\text {Tsir }}} \text { but } E_{2} \not_{\mathrm{B}} E_{\mathcal{I}_{\text {Tsir }}} \text {. }
$$

In both cases their proofs made striking use of ideas from Banach space theory by defining a kind of Polishable ideal analog of the Tsirelson Banach space. This idea has naturally become known as the Tsirelson ideal, and its variations have played an important role in further work by Farah on turbulence.

Conjecture 4 of [12] to the effect that there is no Borel equivalence relation $E$ with $E_{0}<_{\mathrm{B}} E<_{\mathrm{B}} E_{2}$ remains open and very likely true.

5. Dichotomies for turbulence. Knowing that the action of the Banach space $c_{0}$ by translation on $\mathbb{R}^{\mathbb{N}}$ is turbulent and gives rise to an equivalence relation $E_{c_{0}}^{\mathbb{R}^{\mathbb{N}}}$ which is $\leq_{\mathrm{B}}$-incomparable with $E_{2}$ (see Hjorth [9]), it was even speculated at Conjecture 7 of [12] that for any Polish group $G$ and turbulent Polish $G$-space $X$, either

$$
E_{2} \leq E_{G}^{X} \quad \text { or } \quad E_{c_{0}}^{\mathbb{R}^{\mathrm{N}}} \leq_{\mathrm{B}} E_{G}^{X} .
$$

This further gathers plausibility from an observation due to Kechris (for a proof see [9]) that $E_{2} \sim_{\mathrm{B}} E_{\ell^{1}}^{\mathbb{R}^{\mathbb{N}}}$, and thus we might hope that $E_{\ell^{1}}^{\mathbb{R}^{\mathbb{N}}}$ and $E_{c_{0}}^{\mathbb{R}^{\mathbb{N}}}$ would stand like Adam and Eve at the very base of the turbulent equivalence relations.

\section{However:}

THEOREM 5.1 (Oliver). For any equivalence relation $E_{S}$ obtained in the Louveau-Veličković construction from 2.3 we have

$$
E_{S}<_{\mathrm{B}} E_{c_{0}}^{\mathbb{R}^{\mathbb{N}}}
$$

Since these Louveau-Veličković equivalence relations are easily seen to arise from turbulent Polish group actions, we see in particular that there are many incomparable turbulent orbit equivalence relations $<_{\mathrm{B}}$-below $E_{c_{0}}^{\mathbb{R}^{\mathbb{N}}}$.

Not only was Conjecture 7 false as stated, the hope it expressed-that one would have a small basis of turbulent orbit equivalence relations, with at least one member of the basis reducing to any other example of an orbit equivalence relation arising from a turbulent Polish group action-was misguided. Since Mike Oliver's result, Farah has advanced steadily on the structure of the turbulent orbit equivalence relations, and used suitable refinements of the Tsirelson ideal and the Louveau-Veličković examples to refute every structural conjecture we might have ever entertained.

Theorem 5.2 (Farah; see [6]). There is no finite or even countably infinite sequence $E_{G_{0}}^{X_{0}}, E_{G_{1}}^{X_{1}}, \ldots, E_{G_{n}}^{X_{n}}, \ldots$ of orbit equivalence relations arising 
from turbulent actions of Polish groups such that for any other Polish group $H$ and turbulent Polish $H$-space $Y$, there is some $k$ with

$$
E_{G_{k}}^{X_{k}} \leq_{\mathrm{B}} E_{H}^{Y} .
$$

Theorem 5.3 (Farah; see [7]). There is a Polish group $G$ and a turbulent Polish $G$-space $X$ which is above no minimal turbulent orbit equivalence relation; that is to say, for each Polish group $H_{0}$ and turbulent Polish $H_{0}$-space $Y_{0}$ with

$$
E_{H_{0}}^{Y_{0}} \leq_{\mathrm{B}} E_{G}^{X},
$$

we may find a Polish group $H_{1}$ and turbulent Polish $H_{1}$-space $Y_{1}$ with

$$
E_{H_{1}}^{Y_{1}}<_{\mathrm{B}} E_{H_{0}}^{Y_{0}} .
$$

6. What remains open? From the original sequence of conjectures the following remain open:

Conjecture 6.1 (Conjecture 1 of [12]). For $E$ a Borel equivalence relation, either we have $E_{1} \leq_{\mathrm{B}} E$ or there is some Polish group $G$ and Polish $G$-space $X$ with $E \sim_{\mathrm{B}} E_{G}^{X}$.

Conjecture 6.2 (Conjecture 4 of [12]). For $E$ a Borel equivalence relation with $E \leq E_{2}$ we have either $E_{2} \sim_{\mathrm{B}} E$ or $E \leq_{\mathrm{B}} E_{0}$.

Conjecture 6.3 (Conjecture 5 of [12]). For $E$ a Borel equivalence relation of the form $E_{G}^{X}$ for some Polish $G$-space $X$, where $G$ is a closed subgroup of $S_{\infty}$, we have either $E_{3} \leq_{\mathrm{B}} E$ or $E \leq_{\mathrm{B}} E_{\infty}$.

Below we give the proof of the result announced in [12] that this conjecture holds when $E \leq_{\mathrm{B}} E_{G}^{X}$, for $G$ a closed invariantly metrizable subgroup of $S_{\infty}$ and $X$ a Polish $G$-space.

Added in proof. Hjorth has now proved the full Conjecture 6.3. His proof appears in his preprint "A dichotomy theorem for isomorphism".

Conjecture 6.4 (Conjecture 6 of [12]). For $G$ a Polish group and $X$ a Polish $G$-space, either we have some turbulent Polish $G$-space $Y$ with $E_{G}^{Y} \leq{ }_{\mathrm{B}} E_{G}^{X}$ or there is some Polish $S_{\infty}$-space $Z$ with $E_{G}^{X} \sim_{\mathrm{B}} E_{S_{\infty}}^{Z}$.

In particular, these conjectures imply that for any Borel equivalence relation one of the following holds:

(i) $E_{1} \leq_{\mathrm{B}} E$,

(ii) $E_{G}^{X} \leq_{\mathrm{B}} E$ for some turbulent $G$-space $X$,

(iii) $E_{3} \leq_{\mathrm{B}} E$,

(iv) $E \leq_{\mathrm{B}} E_{\infty}$.

This consequence is also open at this time. 
7. The Sixth Dichotomy Theorem. We present here the proof of the following result, labeled the Sixth Dichotomy Theorem in [12].

TheORem 7.1. Let $E$ be a Borel equivalence relation. If $E \leq_{\mathrm{B}} E_{3}$, then

$$
E \leq_{\mathrm{B}} E_{0} \quad \text { or } E \sim_{\mathrm{B}} E_{3} .
$$

As discussed in $\S 11$ of [12] it is enough to prove the following two results.

TheOREM 7.2. Let $E$ be a Borel equivalence relation such that $E \leq_{\mathrm{B}}$ $E_{\infty}^{\mathbb{N}}$. Then exactly one of the following holds:

(i) $E \leq_{\mathrm{B}} E_{\infty}$,

(ii) $E_{3} \sqsubseteq_{\mathrm{c}} E$,

where $\sqsubseteq_{\mathrm{c}}$ means that there is an injective continuous reduction.

TheOREM 7.3. Let $G_{i}, i=1,2, \ldots$, be closed subgroups of $S_{\infty}$, let $G=$ $\prod_{i=1}^{\infty} G_{i}$ and let $X$ be a Borel $G$-space. If $E \leq_{\mathrm{B}} E_{G}^{X}$ and $E \leq_{\mathrm{B}} E_{\infty}$, then for each $n$, there is a Borel $G^{n}$-space $Z_{n}$, where $G^{n}=\prod_{i \leq n} G_{i}$, such that

$$
E \leq_{\mathrm{B}} \bigoplus_{n} E_{G_{n}}^{Z_{n}}
$$

where $\bigoplus$ denotes direct sum.

To see that 7.2 and 7.3 together imply 7.1 , let $E \leq_{\mathrm{B}} E_{3}$. Then $E \leq_{\mathrm{B}}$ $E_{3} \leq_{\mathrm{B}} E_{\infty}^{\mathbb{N}}$, so by 7.1 either $E_{3} \sqsubseteq_{\mathrm{c}} E$, so $E \sim_{\mathrm{B}} E_{3}$, or else $E \leq_{\mathrm{B}} E_{\infty}$. If the last alternative holds, we have $E \leq_{\mathrm{B}} E_{3}=E_{0}^{\mathbb{N}}$ and $E \leq_{\mathrm{B}} E_{\infty}$.

As discussed in [4], $E_{0} \sim_{\mathrm{B}} E_{\mathbb{Z}}^{2^{\mathbb{Z}}}$, where the action of $\mathbb{Z}$ on $2^{\mathbb{Z}}$ is the shift action. So $E \leq_{\mathrm{B}} E_{0}^{\mathbb{N}} \sim_{\mathrm{B}}\left(E_{\mathbb{N}}^{2^{\mathbb{Z}}}\right)^{\mathbb{N}} \sim_{\mathrm{B}} E_{2^{\mathbb{N}}}^{X}$, where $X=\left(2^{\mathbb{Z}}\right)^{\mathbb{N}}$ and $\mathbb{Z}^{\mathbb{N}}$ acts on $\left(2^{\mathbb{N}}\right)^{\mathbb{N}}$ coordinatewise. Since $\mathbb{Z}$ is a closed subgroup of $S_{\infty}, 7.3$ implies that $E$ is Borel reduced to a direct sum of a sequence of equivalence relations of the form $E_{\mathbb{Z}^{n}}^{Y}$. As discussed in [13], it is a theorem of Weiss that any orbit equivalence relation associated with a Borel $\mathbb{Z}^{n}$-space is $\leq_{\mathrm{B}} E_{0}$. So $E \leq_{\mathrm{B}} E_{0}$.

We now present the proofs of 7.2 and 7.3.

Proof of 7.2. Since $E_{\infty}$ can be realized in the form $E_{G}^{2^{\mathrm{N}}}$, where $G$ is a countable group acting continuously on $2^{\mathbb{N}}$, it is enough to prove that (i) or (ii) hold for any $E \leq_{\mathrm{B}}\left(E_{G}^{2^{\mathbb{N}}}\right)^{\mathbb{N}}$, where $G$ is a countable group acting continuously on $2^{\mathbb{N}}$. We can clearly assume that $E$ lives on $X=2^{\mathbb{N}}$. We also claim that we can assume (by changing $G$ to $\mathbb{Z}_{2} \times G$ if necessary) that there is a Borel map $f: X \rightarrow\left(2^{\mathbb{N}}\right)^{\mathbb{N}}$ which is 1-1 and for which there is a continuous $f^{*}:\left(2^{\mathbb{N}}\right)^{\mathbb{N}} \rightarrow X$ such that $f^{*} \mid f[X]=f^{-1}$, and $x E y \Leftrightarrow f(x)\left(E_{G}^{2^{\mathbb{N}}}\right)^{\mathbb{N}} f(y)$. Indeed, if $g: X \rightarrow\left(2^{\mathbb{N}}\right)^{\mathbb{N}}$ is Borel such that $x E y \Leftrightarrow g(x)\left(E_{G}^{2^{\mathbb{N}}}\right)^{\mathbb{N}} g(y)$, define $f: X \rightarrow\left(2^{\mathbb{N}}\right)^{\mathbb{N}}\left(\equiv 2^{\mathbb{N} \times \mathbb{N}}\right.$, via $\left.x_{i}(j)=x(i, j)\right)$ by

$$
f(x)(i, 0)=x(i), \quad f(x)(i, j+1)=g(x)(i, j) .
$$


Then let $\mathbb{Z}_{2} \times G$ act on $2^{\mathbb{N}}$ as follows:

$$
(a, g) * x=(a+x(0))^{\wedge} g \cdot x^{\prime}, \quad \text { where } x^{\prime}(i)=x(i+1)
$$

(here $g \cdot x$ is the given action that defines $E_{G}^{2^{\mathbb{N}}}$ ). Denoting by $E_{\mathbb{Z}_{2} \times G}^{2^{\mathbb{N}}}$ the equivalence relation induced by this action, we clearly have

$$
x E y \Leftrightarrow g(x)\left(E_{G}^{2^{\mathbb{N}}}\right)^{\mathbb{N}} g(y) \Leftrightarrow f(x)\left(E_{\mathbb{Z}_{2} \times G}^{2^{\mathbb{N}}}\right)^{\mathbb{N}} f(y) .
$$

Finally, let $f^{*}$ be defined by

$$
f^{*}(z)(i)=z(i, 0) .
$$

Then $f$ is $1-1$, Borel and reduces $E$ to $\left(E_{\mathbb{Z}_{2} \times G}^{2^{\mathbb{N}}}\right)^{\mathbb{N}}$, and $f^{*}$ is continuous and equal to $f^{-1}$ on $f[X]$.

So fix $G, X, E, f, f^{*}$ as above. By relativization, we can assume that $E, f \in \Delta_{1}^{1}$, and $G$ and the action are recursive.

Notation. • For $x, y \in 2^{\mathbb{N} \times \mathbb{N}}$, let

$$
x_{\leq_{n}}=y_{\leq_{n}} \Leftrightarrow \forall i \leq n\left(x_{i}=y_{i}\right) .
$$

Put

$$
x \equiv_{n} y \Leftrightarrow x\left(E_{G}^{2^{\mathbb{N}}}\right)^{\mathbb{N}} y \& x_{\leq n}=y_{\leq n} .
$$

- For each $n$, let

$$
V^{(n)}=\underbrace{\{1\} \times \ldots \times\{1\}}_{n+1} \times G^{\mathbb{N}} .
$$

Then $V^{(n)}$ is an open subgroup of $G^{\mathbb{N}}$ and so has countable index in $G^{\mathbb{N}}$. Clearly, $\left(E_{G}^{2^{\mathbb{N}}}\right)^{\mathbb{N}}=E_{G^{\mathbb{N}}}^{2^{\mathbb{N}}}$, where $G^{\mathbb{N}}$ acts by the product action on $\left(2^{\mathbb{N}}\right)^{\mathbb{N}} \equiv$ $2^{\mathbb{N} \times \mathbb{N}}$. Moreover, $\left(\equiv_{n}\right)=E_{V^{(n)}}^{2^{\mathbb{N}}}$. It follows that every $E_{G^{\mathbb{N}}}^{2^{\mathbb{N}}}$-class contains only countably many $\equiv_{n}$-classes.

- Let $f[X]=X_{0} \subseteq 2^{\mathbb{N} \times \mathbb{N}}$.

- Let $G=\left\{g_{n}\right\}_{n \in \mathbb{N}}$ (a recursive enumeration), with $g_{0}=1$.

For each $n<k$ and each $p$ define

$$
\begin{aligned}
& \mathcal{A}_{n, k, p}=\left\{A \in \Sigma_{1}^{1}, A \subseteq 2^{\mathbb{N} \times \mathbb{N}}:\right. \\
&\left.\forall x, y \in X_{0} \cap A\left(x \equiv_{n} y \Rightarrow \exists i \leq p\left(g_{i} \cdot x_{k}=y_{k}\right)\right)\right\} .
\end{aligned}
$$

Put

$$
A_{0}=\bigcup_{n} \bigcap_{k>n} \bigcup_{p}\left\{A: A \in \mathcal{A}_{n, k, p}\right\}
$$

Claim 1. $A \in \mathcal{A}_{n, k, p} \Rightarrow \exists B \supseteq A\left(B \in \Delta_{1}^{1} \& B \in \mathcal{A}_{n, k, p}\right)$.

Proof. The property " $A \in \mathcal{A}_{n, k, p}$ " is $\Pi_{1}^{1}$ in the codes.

Claim 2. $A_{0} \in \Pi_{1}^{1}$. 
Proof. By Claim 1,

$$
\begin{aligned}
x \in A_{0} & \Leftrightarrow \exists n \forall k>n \exists p \exists A \in \mathcal{A}_{n, k, p}(x \in A) \\
& \Leftrightarrow \exists n \forall k>n \exists p \exists A \in \Delta_{1}^{1}, A \in \mathcal{A}_{n, k, p}(x \in A) .
\end{aligned}
$$

We now have 2 cases:

CASE I: $X_{0} \subseteq A_{0}$. We will then show that $E \leq_{\Delta_{1}^{1}} F$, where $F$ is $\Delta_{1}^{1}$ and countable. For that it is enough to find a $\Delta_{1}^{1}$ function $g: X \rightarrow Y$, with $Y$ a recursively presented Polish space, so that $g\left([x]_{E}\right)$ is countable and $\neg x E y \Rightarrow g\left([x]_{E}\right) \cap g\left([y]_{E}\right)=\emptyset$ (see, e.g., [10]).

Since $X_{0} \subseteq A_{0}$, we have

$\forall x \in X_{0} \exists n \forall k>n \exists p \exists A \in \Delta_{1}^{1}$

$$
\left(x \in A \& \forall y, z \in X_{0} \cap A\left(y \equiv_{n} z \Rightarrow \exists i \leq p\left(g_{i} \cdot y_{k}=z_{k}\right)\right)\right) .
$$

So there is a $\Delta_{1}^{1}$ function $x \mapsto n(x)$ such that

$$
\begin{aligned}
x \in X_{0} & \Rightarrow \forall k>n(x) \exists p \exists A \in \Delta_{1}^{1} \\
& \left(x \in A \& \forall y, z \in X_{0} \cap A\left(y \equiv_{n} z \Rightarrow \exists i \leq p\left(g_{i} \cdot y_{k}=z_{k}\right)\right)\right) .
\end{aligned}
$$

Let $P_{n}=\{x: n(x)=x\}\left(\in \Delta_{1}^{1}\right)$ and

$$
Q_{n}=P_{n} \cap X_{0} \quad\left(\in \Delta_{1}^{1}\right) .
$$

Clearly, $X_{0}=\bigcup_{n} Q_{n}$ and

$$
\begin{aligned}
x \in Q_{n} & \Rightarrow \forall k>n \exists p \exists A \in \Delta_{1}^{1} \\
& \left(x \in A \& \forall y, z \in X_{0} \cap A\left(y \equiv_{n} z \Rightarrow \exists i \leq p\left(g_{i} \cdot y_{k}=z_{k}\right)\right)\right) .
\end{aligned}
$$

We will find a $\Delta_{1}^{1}$ function $f_{n}: 2^{\mathbb{N} \times \mathbb{N}} \rightarrow 2^{\mathbb{N} \times \mathbb{N}}$ such that

$$
x, y \in Q_{n} \& x \equiv_{n} y \Rightarrow f_{n}(x)=f_{n}(y) \equiv_{n} x .
$$

Then for $x \in X$, let

$$
g(x)=\langle n, y\rangle \Leftrightarrow f(x) \in Q_{n} \& f_{n}(f(x))=y .
$$

This is $\Delta_{1}^{1}$ and we claim that it works: To see that $g\left([x]_{E}\right)$ is countable, just use the fact that there are only countably many $\equiv_{n}$-classes in each $E_{G^{\mathbb{N}}}^{2^{\mathbb{N}}}$-class. Now assume $g(x)=g(y)=\langle n, z\rangle$. Then $f(x), f(y) \in Q_{n}$ and $f_{n}(f(x))=f_{n}(f(y))(=z)$, so $f(x) \equiv_{n} f(y)$, thus $f(x) E_{G^{\mathbb{N}}}^{2^{\mathbb{N} \times \mathbb{N}}} f(y)$, and so $x E y$.

We will now construct the $f_{n}$ 's:

Fix a $\equiv_{n} \mid Q_{n}$-class $C$. Note that for any $x \in C$ and any $k>n$, there are $p, A \in \Delta_{1}^{1}$ with $x \in A$ and

$$
\forall y, z \in X_{0} \cap A\left(y \equiv_{n} z \Rightarrow \exists i \leq p\left(g_{i} \cdot y_{k}=z_{k}\right)\right) .
$$

So for each $k>n$ let $p_{k}, A_{k}$ be least such that $A_{k} \cap C \neq \emptyset$ and $\forall y, z \in X_{0} \cap A_{k}$ $\left(y \equiv_{n} z \Rightarrow \exists i \leq p_{k}\left(g_{i} \cdot y_{k}=z_{k}\right)\right)$. Then it is clear that $\left\{x_{k}: x \in C \cap A_{k}\right\}$ 
is finite, so let $a_{k}^{C}$ be its least member (in the lexicographical ordering on $\left.2^{\mathbb{N} \times \mathbb{N}} \equiv 2^{\mathbb{N}}\right)$.

Define $\alpha_{C}$ by

$$
\begin{array}{ll}
\left(\alpha_{C}\right)_{i}=x_{i} & \text { for any (all) } x \in C, \text { if } i \leq n ; \\
\left(\alpha_{C}\right)_{k}=a_{k}^{C} & \text { for } k>n .
\end{array}
$$

Then $\alpha_{C} \equiv_{n} x$ for any $x \in C$.

For $x \in Q_{n}$, put

$$
h_{n}(x)=\alpha_{[x]_{\equiv_{n} \mid Q_{n}}} .
$$

Then $h_{n}$ is $C$-measurable (where $C$ is the smallest $\sigma$-algebra containing the open sets and closed under the Suslin operation $\mathcal{A}$ ) and

$$
x, y \in Q_{n} \& x \equiv_{n} y \Rightarrow h_{n}(x)=h_{n}(y) \equiv_{n} x .
$$

Let $\widetilde{Q}_{n}=\left[Q_{n}\right]_{\equiv_{n}}=$ the saturation of $Q_{n}$ by $\equiv_{n}$ in $2^{\mathbb{N} \times \mathbb{N}}$. Clearly, $\widetilde{Q}_{n} \in$ $\Sigma_{1}^{1}$ and there is $q_{n}: \widetilde{Q}_{n} \rightarrow Q_{n}$ which is $C$-measurable with $q_{n}(x) \equiv_{n} x$. If $r_{n}=h_{n} \circ q_{n}$, then $r_{n}: \widetilde{Q}_{n} \rightarrow 2^{\mathbb{N} \times \mathbb{N}}$ is $C$-measurable and

$$
x, y \in \widetilde{Q}_{n} \& x \equiv_{n} y \Rightarrow r_{n}(x)=r_{n}(y) \equiv_{n} x .
$$

Thus $\mu\left(\widetilde{Q}_{n}\right)=0$ for every $\equiv_{n}$-ergodic, non-atomic probability Borel measure on $2^{\mathbb{N} \times \mathbb{N}}$. Since this is a $\Pi_{1}^{1}$ in the codes property of $\widetilde{Q}_{n}$, there is a $\Delta_{1}^{1}$ set $R_{n} \supseteq \widetilde{Q}_{n}, R_{n} \in \Delta_{1}^{1}$, which still has this property. Let

$$
\left\langle R_{n}\right\rangle=\left\{x: \forall y \equiv_{n} x\left(y \in R_{n}\right)\right\} .
$$

Then $\left\langle R_{n}\right\rangle \in \Pi_{1}^{1},\left\langle R_{n}\right\rangle$ is $\equiv_{n}$-invariant, and $\widetilde{Q}_{n} \subseteq\left\langle R_{n}\right\rangle \subseteq R_{n}$, so there is a $\Delta_{1}^{1}, \equiv_{n}$-invariant set $S_{n}$ with $\widetilde{Q}_{n} \subseteq S_{n} \subseteq\left\langle R_{n}\right\rangle$. Then $\mu\left(S_{n}\right)=0$ for any measure as above, so $\equiv_{n} \mid S_{n}$ is smooth. Since $\equiv_{n} \mid S_{n}$ is induced by a $\Delta_{1}^{1}$ action of the Polish group $V^{(n)}$, it follows that $\equiv_{n} \mid S_{n}$ has a $\Delta_{1}^{1}$-selector, i.e., there is a $\Delta_{1}^{1}$ map $f_{n}: 2^{\mathbb{N} \times \mathbb{N}} \rightarrow 2^{\mathbb{N} \times \mathbb{N}}$ such that

$$
x, y \in S_{n} \& x \equiv_{n} y \Rightarrow f_{n}(x)=f_{n}(y) \equiv_{n} x,
$$

and since $Q_{n} \subseteq S_{n}$ we are done.

CASE II: $X_{0} \nsubseteq A_{0}$. We will then show that $E_{0}^{\mathbb{N}} \sqsubseteq_{c} E$. Notice that it is enough to show that $E_{0}^{\mathbb{N}} \sqsubseteq_{\mathrm{c}} E_{0}^{\mathbb{N}} \mid X_{0}$, say by a continuous embedding $e$, because then $f^{*} \circ e$ is a continuous embedding of $E_{0}^{\mathbb{N}}$ into $E$.

Let $Y_{0}=X_{0} \backslash A_{0}$. Then $Y_{0} \neq \emptyset, Y_{0} \in \Sigma_{1}^{1}$. By definition, for any $x \in Y_{0}$,

$$
\forall n \exists k>n \forall p \forall A \in \Sigma_{1}^{1}\left(x \in A \Rightarrow A \notin \mathcal{A}_{n, k, p}\right),
$$

i.e., if $x \in Y_{0}$, then

$$
\begin{aligned}
\forall n \exists k>n & \forall p \forall A \in \Sigma_{1}^{1} \\
& \left(x \in A \Rightarrow \exists y, z \in X_{0} \cap A\left(y \equiv_{n} z \& \forall i \leq p\left(g_{i} \cdot y_{k} \neq z_{k}\right)\right)\right) .
\end{aligned}
$$


Let, for $n<k$,

$$
\begin{aligned}
Y_{n, k} & =\left\{x \in Y_{0}: \forall p \forall A \in \Sigma_{1}^{1}\left(x \in A \Rightarrow A \notin \mathcal{A}_{n, k, p}\right)\right\} \\
& =\left\{x \in Y_{0}: \forall p \forall A \in \Delta_{1}^{1}\left(x \in A \Rightarrow A \notin \mathcal{A}_{n, k, p}\right)\right\}
\end{aligned}
$$

(by Claim 1), so that $Y_{n, k} \in \Sigma_{1}^{1}$. Thus

$$
\forall x \in Y_{0} \forall n \exists k>n\left(x \in Y_{n, k}\right) .
$$

Notation. Below $\langle m, j\rangle$ denotes the usual Cantor bijection of $\mathbb{N} \times \mathbb{N}$ with $\mathbb{N}$, given by

$$
\langle m, j\rangle=(m+j)(m+j+1) / 2+j .
$$

Let $L(n)=\max \{k: \exists i(\langle k, i\rangle \leq n)\}$. Note that $L(\langle m, 0\rangle)=m$ and $L(n) \leq L(n+1)$, while $L(n)=L(n-1)$ if $n=\langle m, j\rangle$ with $j>0$, and $L(n)=L(n-1)+1$ if $n=\langle m, 0\rangle>0$.

We will define the following by induction on $n \geq 0$ :

(i) Non-empty $\Sigma_{1}^{1}$ sets $A_{s}, s \in 2^{n+1}$. These will be chosen so that $A_{\emptyset}=$ $Y_{0}, A_{s \wedge i} \subseteq A_{s}, \operatorname{diam}\left(A_{s}\right) \leq 2^{-\operatorname{lh}(s)}$, and for each $x \in 2^{\mathbb{N}}, A_{x \mid i}$ "converges" in the Gandy-Harrington topology, so that $\bigcap_{i} A_{x \mid i}=\left\{\alpha_{x}\right\}$ and if $\alpha_{i} \in A_{x \mid i}$, then $\alpha_{i} \rightarrow \alpha_{x}$ in the usual topology.

(ii) $k_{m} \in \mathbb{N}, m \leq L(n)$. These will be chosen so that $0<k_{0}<k_{1}<\ldots$

(iii) We will also have

$$
A_{0^{n+1}} \subseteq \bigcap_{r \leq L(n)} Y_{r, k_{r}} .
$$

(iv) $g_{s} \in G^{\mathbb{N}}, s \in 2^{n+1}$, such that $g_{0^{n+1}}=1,\left(g_{s}\right)_{i}=1$ if $i>k_{L(n)}$. (Thus, essentially, $g_{s} \in G^{k_{L(n)}+1}$.)

(v) Links. We will also have, for $s \in 2^{n+1}$,

$$
\forall x \in A_{0^{n+1}} \exists y \in A_{s}\left(g_{s} \cdot x \equiv_{k_{L(n)}} y\right) .
$$

(vi) Positive requirements. For $s, t \in 2^{n+1}$, put $g_{s, t}=g_{t} \cdot g_{s}^{-1}$. If $\bar{n}<n,(\bar{s}, \bar{t}) \subseteq(s, t), \bar{s}, \bar{t} \in 2^{\bar{n}+1}$, then for $l \leq L(n)$ we must have

$$
[\forall \bar{l} \leq l \forall\langle\bar{l}, i\rangle \in(n+1) \backslash(\bar{n}+1)(s(\langle\bar{l}, i\rangle)=t(\langle\bar{l}, i\rangle))] \Rightarrow g_{s, t} \equiv_{l} g_{\bar{s}, \bar{t}},
$$

where for $g, h \in G^{\mathbb{N}}, l \in \mathbb{N}$ we let

$$
g \equiv_{l} h \Leftrightarrow \forall i \leq l\left(g_{i}=h_{i}\right) .
$$

(vii) Negative requirements. If $s, t \in 2^{n+1}, n=\langle m, j\rangle$, then we must have

$$
s(n) \neq t(n) \Rightarrow \forall l \leq n\left(x \in A_{s} \& y \in A_{t} \Rightarrow g_{l} \cdot x_{k_{m}} \neq y_{k_{m}}\right) .
$$

Assume all this can be done. Then we claim that

$$
x E_{0}^{\mathbb{N}} y \Leftrightarrow \alpha_{x} E_{G^{\mathbb{N}}}^{2^{\mathbb{N}}} \alpha_{y},
$$


which, since $\alpha_{x} \in Y_{0}$, proves what we want. (Notice that by (vii), $A_{s \wedge 0} \cap$ $A_{s^{\wedge} 1}=\emptyset$ (as $\left.g_{0}=1\right)$, so $x \mapsto \alpha_{x}$ is 1-1 and clearly continuous.)

Proof of $(*) . \Rightarrow$ Assume $x E_{0}^{\mathbb{N}} y$. Fix $l$. Choose $t_{0}, t_{1}, \ldots, t_{l}$ such that

$$
x(\langle\bar{l}, i\rangle)=y(\langle\bar{l}, i\rangle) \quad \text { for } \bar{l} \leq l \text { and } i \geq t_{\bar{l}} .
$$

Then there is $\bar{n}$ with $L(\bar{n}) \geq l$ such that for any $n>\bar{n}$ we have

$$
\forall \bar{l} \leq l \forall\langle\bar{l}, i\rangle \in(n+1) \backslash(\bar{n}+1)[x|(n+1)(\langle\bar{l}, i\rangle)=y|(n+1)(\langle\bar{l}, i\rangle)] .
$$

So, by (vi) for $n>\bar{n}$, we have

$$
g_{x|(n+1), y|(n+1)} \equiv_{l} g_{x|(\bar{n}+1), y|(\bar{n}+1)} .
$$

By (v), find $w_{x \mid(n+1)}, w_{y \mid(n+1)}$ in $A_{x \mid(n+1)}, A_{y \mid(n+1)}$, respectively, so that

$$
g_{x \mid(n+1)} \cdot \alpha_{0 \infty} \equiv_{k_{L(n)}} w_{x \mid(n+1)}, \quad g_{y \mid(n+1)} \cdot \alpha_{0 \infty} \equiv_{k_{L(n)}} w_{y \mid(n+1)} .
$$

Thus

$$
g_{y \mid(n+1)} g_{x \mid(n+1)}^{-1} \cdot w_{x \mid(n+1)} \equiv_{k_{L(n)}} w_{y \mid(n+1)},
$$

and so, since $L(n) \geq L(\bar{n}) \geq l$ and therefore $k_{L(n)} \geq l$, we have

$$
g_{x|(n+1), y|(n+1)} \cdot w_{x \mid(n+1)} \equiv_{l} w_{y \mid(n+1)} .
$$

Hence

$$
g_{x|(\bar{n}+1), y|(\bar{n}+1)} \cdot w_{x \mid(n+1)} \equiv_{l} w_{y \mid(n+1)} .
$$

Taking the limit as $n \rightarrow \infty$, we get

$$
\left(g_{x|(\bar{n}+1), y|(\bar{n}+1)} \cdot \alpha_{x}\right)_{\bar{l}}=\left(\alpha_{y}\right)_{\bar{l}}, \quad \forall \bar{l} \leq l,
$$

so, in particular, there is $g_{l} \in G$ with $g_{l} \cdot\left(\alpha_{x}\right)_{l}=\left(\alpha_{y}\right)_{l}$. Since this is true for every $l$, we see that $\alpha_{x} E_{G^{\mathbb{N}}}^{2^{\mathbb{N} \times \mathbb{N}}} \alpha_{y}$.

$\Leftarrow$ Assume $\neg x E_{0}^{\mathbb{N}} y$. Fix $m$ such that $x(\langle m, j\rangle) \neq y(\langle m, j\rangle)$ for infinitely many $j$. Assume, towards a contradiction, that $\alpha_{x} E_{G^{\mathbb{N}}}^{2^{\mathbb{N}}} \alpha_{y}$. Let $l$ be such that $g_{l} \cdot\left(\alpha_{x}\right)_{k_{m}}=\left(\alpha_{y}\right)_{k_{m}}$. Fix $n=\langle m, j\rangle$ such that $x(n) \neq y(n)$ and $n \geq l$. Then $x|(n+1)(n) \neq y|(n+1)(n)$, so by (vi), as $\alpha_{x} \in A_{x \mid(n+1)}, y \in A_{y \mid(n+1)}$, we have

$$
g_{l} \cdot\left(\alpha_{x}\right)_{k_{m}} \neq\left(\alpha_{y}\right)_{k_{m}}
$$

a contradiction.

\section{CONSTRUCTION}

SteP 1. Let $y \in Y_{0}$. Then $\forall k \exists n>k\left(y \in Y_{n, k}\right)$, so fix $k_{0}>0$ such that $y \in Y_{0, k_{0}}$, and so $\forall p \forall A \in \Sigma_{1}^{1}\left(y \in A \Rightarrow A \notin \mathcal{A}_{0, k_{0}, p}\right)$. Recall that $g_{0}=1$. So for $p=0$ and $A=Y_{0, k_{0}}$ we have $Y_{0, k_{0}} \notin \mathcal{A}_{0, k_{0}, 0}$, so there are $x_{(0)}, x_{(1)} \in Y_{0, k_{0}}$ with $x_{(0)} \equiv_{0} x_{(1)}$, but $g_{0} \cdot\left(x_{(0)}\right)_{k_{0}}=\left(x_{(0)}\right)_{k_{0}} \neq\left(x_{(1)}\right)_{k_{0}}$. Let 
$g_{(0)}=1$ and let $g_{(1)}^{\prime} \in G^{\mathbb{N}}$ be such that $g_{(1)}^{\prime} \cdot x_{(0)}=x_{(1)}$. Define $g_{(1)} \in G^{\mathbb{N}}$ by

$$
\left(g_{(1)}\right)_{i}= \begin{cases}\left(g_{(1)}^{\prime}\right)_{i} & \text { if } i \leq k_{0}=k_{L(0)}, \\ 1 & \text { if } i>k_{0} .\end{cases}
$$

Then $g_{(1)} \cdot x_{(0)} \equiv_{k_{0}} x_{(1)}$. Let then $A_{(0)}, A_{(1)}$ be small enough $\Sigma_{1}^{1}$ subsets of $Y_{0, k_{0}}\left(\subseteq Y_{0}=A_{\emptyset}\right)$ so that the following are satisfied: (i), (ii) $\left(k_{0}>0\right)$, (iii) $\left(A_{(0)} \subseteq Y_{0, k_{0}}\right)$, (iv), (v), (vi) (vacuously), (vii) (if $\left(x_{(0)}\right) k_{0}(t) \neq\left(y_{(0)}\right) k_{0}(t)$, we just make sure that every $x \in A_{(0)}$ agrees with $x_{(1)}$ at $\left.\left(k_{0}, t\right)\right)$.

STEP $n+1(n>0)$. Assume the construction has been done up to level $n$, i.e., for $\bigcup_{k \leq n} 2^{n}$, and $k_{m}$ has been defined for $m \leq L(n-1)$. We now consider $2^{n+1}$. Let $n=\langle m, j\rangle$. We consider two cases, (A) and (B).

(A) $j>0$. Then $L(n)=L(n-1)$, so $k_{m}$ is already defined for all $m \leq L(n)$.

First we shrink all $A_{s}, s \in 2^{n}$, to make sure that $\operatorname{diam}\left(A_{s}\right)<2^{-\operatorname{lh}(s)-1}$, and "convergence" in the Gandy-Harrington topology is improved, and moreover, this is done so that (v) still remains valid (for $s \in 2^{n}$ of course). To avoid complicated notation we will still call these smaller sets $A_{s}$ (we have not changed, by the way, the $\left.g_{s}, s \in 2^{n}\right)$. So all conditions (i)-(vii) are satisfied up to that point, and we took care of (i) at Step $n+1$. Also, as we pointed out, (ii) has been taken care of at Step $n+1$, and we have

$$
A_{0^{n}} \subseteq \bigcap_{r \leq L(n-1)} Y_{r, k_{r}}=\bigcap_{r \leq L(n)} Y_{r, k_{r}} .
$$

So choose $x_{0^{n+1}}, x_{0^{n \wedge}} \in A_{0^{n}}$ so that $x_{0^{n+1}} \equiv_{m} x_{0^{n \wedge}}($ as $m \leq L(n))$ and $g_{s_{1}}^{-1} g_{l}^{ \pm 1} g_{t_{1}} \cdot\left(x_{0^{n+1}}\right)_{k_{m}} \neq\left(x_{0^{n \wedge}}\right)_{k_{m}}$ for all $l \leq n$ and all $s_{1}, t_{1} \in 2^{n}$. This is possible since $A_{0^{n}} \subseteq Y_{m, k_{m}}$. Let $g_{0^{n+1}}=1$ and $g_{0^{n} \wedge}^{\prime} \in G^{\mathbb{N}}$ be such that $g_{0^{n} \wedge}^{\prime} \cdot x_{0^{n+1}}=x_{0^{n} \wedge}$. As $x_{0^{n+1}} \equiv_{m} x_{0^{n \wedge} 1}$, we can assume that $\left(g_{0^{n}{ }_{1}}^{\prime}\right)_{i}=1$ for $i \leq m$.

Define $g_{0^{n} \wedge}$ by

$$
\left(g_{0^{n \wedge} 1}\right)_{i}= \begin{cases}\left(g_{0^{n \wedge}}^{\prime}\right)_{i} & \text { for } i \leq k_{L(n)}, \\ 1 & \text { for } i>k_{L(n)} .\end{cases}
$$

Then for $s \in 2^{n+1}, s=\bar{s}^{\wedge} i$ define $g_{s}$ by

$$
g_{s}= \begin{cases}g_{\bar{s}} & \text { if } i=0 \\ g_{\bar{s}} g_{0^{n \wedge} 1} & \text { if } i=1 .\end{cases}
$$

(Notice that this is consistent with the previous definitions of $g_{0^{n+1}}, g_{0^{n} \wedge}$.) Then (iv) is clearly satisfied, as $L(n)=L(n-1)$.

We next verify (vi).

Take $s, t \in 2^{n+1}$, say $s=s_{1} \wedge i, t=t_{1}^{\wedge} j$. If $i=j$ then $g_{s, t}=g_{s_{1}, t_{1}}$. Moreover, as $g_{0^{n} \wedge} \equiv_{m} 1$, we always clearly have $g_{s, t} \equiv_{m} g_{s_{1}, t_{1}}$. 
Now consider $\bar{n}<n,(\bar{s}, \bar{t}) \subseteq(s, t), \bar{s}, \bar{t} \in 2^{\bar{n}+1}$ and fix $l$ such that

$$
\forall \bar{l} \leq l \forall\langle\bar{l}, i\rangle \in(n+1) \backslash(\bar{n}+1)(s(\langle\bar{l}, i\rangle)=t(\langle\bar{l}, i\rangle)) .
$$

CASE $1: i=j$. If $\bar{n}=n-1$, then $\bar{s}=s_{1}, \bar{t}=t_{1}$ and $g_{s, t}=g_{s_{1}, t_{1}}=g_{\bar{s}, \bar{t}}$, so we are done. If $\bar{n}<n-1$, then, by induction hypothesis, $g_{s_{1}, t_{1}} \equiv_{l} g_{\bar{s}, \bar{t}}$, so $g_{s, t}\left(=g_{s_{1}, t_{1}}\right) \equiv_{l} g_{\bar{s}, \bar{t}}$.

CASE 2: $i \neq j$. Then we must have $l<m$. Since $g_{s, t} \equiv_{m} g_{s_{1}, t_{1}}$, and thus $g_{s, t} \equiv_{l} g_{s_{1}, t_{1}}$, we are done as in Case 1 .

Since $x_{0^{n+1}}, x_{0^{n} \wedge} \in A_{0^{n}}$, by induction hypothesis $((\mathrm{v})$-recall that $L(n)=L(n-1))$ we can find $x_{s_{1} \wedge} \in A_{s_{1}}$ so that $g_{s_{1}} \cdot x_{0^{n+1}} \equiv_{k_{L(n)}}$ $x_{s_{1} \wedge 0}\left(s_{1} \in 2^{n}\right)$ and $x_{s_{1} \wedge 1} \in A_{s_{1}}$ so that $g_{s_{1}} \cdot x_{0^{n} \wedge} \equiv_{k_{L(n)}} x_{s_{1} \wedge 1}\left(s_{1} \in 2^{n}\right)$. (Again $x_{0^{n+1}}, x_{0^{n \wedge}}$ are consistently defined.) Then $g_{s_{1} \wedge 0} \cdot x_{0^{n+1}} \equiv_{k_{L(n)}} x_{s_{1} \wedge}$ and

$$
g_{s_{1} \wedge} \cdot x_{0^{n+1}}=g_{s_{1}} \cdot\left(g_{0^{n} \wedge} \cdot x_{0^{n+1}}\right)=g_{s_{1}} \cdot x_{0^{n \wedge}} \equiv_{k_{L(n)}} x_{s_{1} \wedge} .
$$

Next notice that, as $k_{m} \leq k_{L(n)}$, we have $\left(g_{s_{1} \wedge 0}\right)_{k_{m}} \cdot\left(x_{0^{n+1}}\right)_{k_{m}}=\left(x_{s_{1} \wedge 0}\right)_{k_{m}}$ and $\left(x_{t_{1} 1}\right)_{k_{m}}=\left(g_{t_{1}}\right)_{k_{m}} \cdot x_{0^{n^{\wedge}}{ }^{1}}$, so we cannot have $g_{l} \cdot\left(x_{s_{1} \wedge}\right)_{k_{m}}=\left(x_{t_{1} \wedge 1}\right)_{k_{m}}$, and similarly we cannot have $g_{l} \cdot\left(x_{s_{1} \wedge 1}\right)_{k_{m}}=\left(x_{t_{1} \wedge 0}\right)_{k_{m}}$, for any $l \leq n$ and any $s_{1}, t_{1} \in 2^{n}$. Clearly, this conclusion can be guaranteed by fixing only finitely many values of $\left(x_{s}\right)_{k_{m}}, s \in 2^{n+1}$.

Thus it is routine to define $A_{s} \in \Sigma_{1}^{1}$ with $x_{s} \in A_{s}, s \in 2^{n+1}$, so that all conditions (i)-(vii) are satisfied (for (vii) we just make sure that all $x \in A_{s}$ agree with $x_{s}$ on enough, but finitely many, values).

(B) $j=0$. Then $L(n)=L(n-1)+1=m$.

Again we may assume that we have shrunk the $A_{s}, s \in 2^{n}$, so that (i) will be satisfied at level $n+1$. Next fix $y_{0} \in A_{0^{n}}$. Then $y_{0} \in Y_{0}$, so $\forall n^{\prime} \exists k^{\prime}>n^{\prime}\left(y_{0} \in Y_{n^{\prime}, k^{\prime}}\right)$, and by taking $n^{\prime}=k_{m-1}=k_{L(n-1)}$ we can find $k_{m}=k^{\prime}>k_{m-1}$ with $y_{0} \in Y_{k_{m-1}, k_{m}}$, so, in particular, $y_{0} \in Y_{m, k_{m}}$ as $k_{m-1} \geq m$. Hence, by shrinking again if necessary, we can assume that $A_{0^{n}} \subseteq \bigcap_{r \leq L(n)} Y_{r, k_{r}}=\bigcap_{r \leq L(n-1)} Y_{r, k_{r}} \cap Y_{m, k_{m}}$. Next choose $x_{s} \in A_{s}$, $s \in 2^{n}$, so that $g_{s} \cdot x_{0^{n}} \equiv_{k_{L(n-1)}} x_{s}$, and fix $\bar{g}_{s} \in G^{\mathbb{N}}$ so that $g_{s} \equiv_{k_{L(n-1)}} \bar{g}_{s}$, $\left(\bar{g}_{s}\right)_{i}=0$ if $i>k_{L(n)}$ and $\bar{g}_{s} \cdot x_{0^{n}} \equiv_{k_{L(n)}} x_{s}$. Then, as $g_{s} \equiv_{k_{L(n-1)}} \bar{g}_{s}$, it is clear that (vi) is still satisfied if (for $s \in 2^{n}$ ) we replace $g_{s}$ by $\bar{g}_{s}$. It follows that we can shrink $A_{s}, s \in 2^{n}$, to $\bar{A}_{s}, s \in 2^{n}$, so that all of (i)-(vii) are satisfied with $\bar{A}_{s}, \bar{g}_{s}$ replacing $A_{s}, g_{s}$, and moreover, $\bar{A}_{s}$ satisfies (i) for level $n+1$, $\bar{A}_{0^{n+1}} \subseteq \bigcap_{r \leq L(n)} Y_{r, k_{r}},\left(\bar{g}_{s}\right)_{i}=0$ for all $i>k_{L(n)}$, and $\forall x \in \bar{A}_{0^{n}} \exists y \in \bar{A}_{s}$ $\left(\bar{g}_{s} \cdot x \equiv_{k_{L(n)}} y\right)$. So, to avoid complicated notation, we may as well assume that at step $n$ we already have $A_{s}, g_{s}\left(s \in 2^{n}\right)$ satisfying all these conditions. But then we can repeat exactly the construction of the previous case (A).

Proof of 7.3. We will first deal with the case $E=E_{G}^{X}$, which is simpler. For each $n$ fix a clopen basis $\left\{U_{k}^{n}\right\}_{k \in \mathbb{N}}$ for $G^{n}$ closed under right multiplica- 
tion. Identifying $U_{k}^{n}$ with $U_{k}^{n} \times G_{n+1} \times G_{n+2} \times \ldots$, we derive that $\left\{U_{k}^{n}\right\}_{k \in \mathbb{N}}$ is closed under multiplication by elements of $G$ and $\left\{U_{k}^{n}\right\}_{n, k \in \mathbb{N}}$ is a basis for $G$.

If $E_{\infty}$ lives on $Y$, let $h$ be a Borel function witnessing that $E_{G}^{X} \leq_{\mathrm{B}} E_{\infty}$. Fix $x \in X$, and let $[h(x)]_{E_{\infty}}=\left\{y_{i}\right\}_{i \in \mathbb{N}}$. Then $G=\bigcup_{i}\left\{g: h(g \cdot x)=y_{i}\right\}$, so for some $i,\left\{g: h(g \cdot x)=y_{i}\right\}$ is non-meager, thus comeager in some $U_{k}^{n}$. So $\forall x \exists n P(x, n) \quad$ with $P(x, n) \Leftrightarrow \exists k \exists y \in[h(x)]_{E_{\infty}} \forall^{*} g \in U_{k}^{n}(h(g \cdot x)=y)$.

Clearly, $P$ is Borel. Moreover, it is $E_{G}^{X}$-invariant, i.e., if $P(x, n) \& x^{\prime} E_{G}^{X} x$, then $P\left(x^{\prime}, n\right)$. To see this, let $x^{\prime}=g_{0}^{-1} \cdot x$. Then

$$
\exists k \exists y \in[h(x)]_{E_{\infty}} \forall^{*} g \in U_{k}^{n}\left(h\left(g g_{0} \cdot x^{\prime}\right)=y\right),
$$

SO

$$
\exists k \exists y \in\left[h\left(x^{\prime}\right)\right]_{E_{\infty}} \forall^{*} g^{\prime} \in U_{k}^{n} g_{0}\left(h\left(g^{\prime} \cdot x^{\prime}\right)=y\right) .
$$

Since $U_{k}^{n} g_{0}=U_{k^{\prime}}^{n}$ for some $k^{\prime}$, we see that $P\left(x^{\prime}, n\right)$ holds. Thus, by the invariant uniformization theorem, there is an $E_{G}^{X}$-invariant Borel function $F: X \rightarrow \mathbb{N}$ with $P(x, F(x)), \forall x \in X$. Let $X_{n}=F^{-1}(\{n\})$. Then $X_{n}$ is an $E_{G}^{X}$-invariant Borel set. We will find a Borel $G^{n}$-space $Y_{n}$ so that $E_{G}^{X} \mid X_{n} \leq{ }_{\mathrm{B}} E_{G^{n}}^{Y_{n}}$, which will complete the proof.

Fix $\infty \notin Y$ and let

$$
Y_{n}=(Y \oplus\{\infty\})^{\left\{U_{k}^{n}\right\}_{k \in \mathbb{N}}} .
$$

Then have $G^{n}$ act on $Y_{n}$ by right-shift, i.e.,

$$
g \cdot H\left(U_{k}^{n}\right)=H\left(U_{k}^{n} g\right) .
$$

Clearly, this is a Borel action. Now define $Q_{n}: X_{n} \rightarrow Y_{n}$ as follows: $Q_{n}(x)\left(U_{k}^{n}\right)=$ the unique $y \in[h(x)]_{E_{\infty}}$ such that $\forall^{*} g \in U_{k}^{n}(h(g \cdot x)=y)$, if such exists; $\infty$ otherwise. Clearly, $Q_{n}$ is Borel. We verify that for $x, x^{\prime} \in X_{n}$,

$$
x E_{G}^{X} x^{\prime} \Leftrightarrow Q(x) E_{G_{n}}^{Y_{n}} Q(y) .
$$

$\Rightarrow$ Say $g_{0} \cdot x=x^{\prime}$. Let $g_{0}=\left(g_{1}, g_{2}, \ldots\right)$ and $g^{n}=\left(g_{1}, \ldots, g_{n}\right)$. We will check that $g^{n} \cdot Q_{n}(x)=Q_{n}\left(x^{\prime}\right)$, i.e., $Q_{n}(x)\left(U_{k}^{n} g^{n}\right)=Q\left(x^{\prime}\right)\left(U_{k}^{n}\right)$. Indeed, for $y \in[h(x)]_{E_{\infty}}=\left[h\left(x^{\prime}\right)\right]_{E_{\infty}}$, we have $Q(x)\left(U_{k}^{n} g^{n}\right)=y$ iff $\forall^{*} g^{\prime} \in U_{k}^{n}$ $\left(h\left(g^{\prime} \cdot x^{\prime}\right)=y\right)$ iff $Q\left(x^{\prime}\right)\left(U_{k}^{n}\right)=y$.

$\Leftarrow$ Now assume that $Q(x) E_{G^{n}}^{Y_{n}} Q\left(x^{\prime}\right)$. Since $x \in X_{n}$, fix $k$ so that $\forall^{*} g \in$ $U_{k}^{n}(h(g \cdot x)=y)$, for some $y \in[h(x)]_{E_{\infty}}$. Thus $Q(x)\left(U_{k}^{n}\right)=y$. Let $g^{n} \in G^{n}$ be such that $g^{n} \cdot Q(x)=Q\left(x^{\prime}\right)$. Then $Q\left(x^{\prime}\right)\left(U_{l}^{n}\right)=Q(x)\left(U_{l}^{n} g^{n}\right)$ for any $l$, so if $l$ is so chosen that $U_{k}^{n}=U_{l}^{n} g^{n}$, we have $Q\left(x^{\prime}\right)\left(U_{l}^{n}\right)=Q(x)\left(U_{k}^{n}\right)=y$, thus $y \in\left[h\left(x^{\prime}\right)\right]_{E_{\infty}}$ and $h(x) E_{\infty} h\left(x^{\prime}\right)$, and $x E_{G}^{X} x^{\prime}$.

Now consider the general case. Assume that $E$ lives on $Z$ and that the functions $f, h$ witnessing, resp., that $E \leq_{\mathrm{B}} E_{G}^{X}$ and $E \leq_{\mathrm{B}} E_{\infty}$ are continu- 
ous. Fix a basis $\left\{W_{m}\right\}$ for $Z$ and a basis $\left\{N_{p}\right\}$ for $Y$. Put

$$
P(x, g, z, y) \Leftrightarrow f(z)=g \cdot x \& h(z)=y .
$$

This is closed in $X \times G \times Z \times Y$. For each $x \in[f(z)]_{E_{G}^{X}}, \operatorname{proj}_{Y}(P(x))$ is $\boldsymbol{\Sigma}_{1}^{1}$, non-empty and countable, so there are $n, k, m, p$ such that $\operatorname{proj}_{Y}(P(x) \cap$ $\left.\left(U_{k}^{n} \times W_{m} \times N_{p}\right)\right)$ is a singleton, say $y \in[h(x)]_{E_{\infty}}$. Then for $N_{q} \subseteq N_{p}$,

$$
y \in N_{q} \Leftrightarrow \operatorname{proj}_{Y}\left(P(x) \cap\left(U_{k}^{n} \times W_{m} \times N_{q}\right)\right) \neq \emptyset .
$$

Put

$$
R(x, n, k, m, p) \Leftrightarrow \operatorname{proj}_{Y}\left(P(x) \cap\left(U_{k}^{n} \times W_{m} \times N_{p}\right)\right)=\emptyset .
$$

This is $\boldsymbol{\Pi}_{1}^{1}$ and invariant under the $\boldsymbol{\Sigma}_{1}^{1}$ equivalence relation

$$
\begin{aligned}
& (x, n, k, m, p) \sim\left(x^{\prime}, n^{\prime}, k^{\prime}, m^{\prime}, p^{\prime}\right) \\
& \quad \Leftrightarrow n=n^{\prime}, m=m^{\prime}, p=p^{\prime} \& \exists g_{0}\left(g_{0}^{-1} \cdot x=x^{\prime} \& U_{k}^{n} g_{0}=U_{k^{\prime}}^{n}\right) .
\end{aligned}
$$

So, by Solovay's Theorem (see [14], 34.6(ii)), there is an $\sim$-invariant $\Pi_{1}^{1}$-rank $\varphi: R \rightarrow \omega_{1}$ on $R$. As usual, let $\varphi=\omega_{1}$ off $R$. We have

$\forall z \exists y \in[h(z)]_{E_{\infty}} \exists n, k, m, p\left[y \in N_{p} \& \forall q\left(N_{q} \subseteq N_{p} \Rightarrow\right.\right.$

- $y \in N_{q} \Rightarrow \varphi(f(z), n, k, m, q)=\omega_{1}$,

- $\left.\left.y \notin N_{q} \Rightarrow \varphi(f(z), n, k, m, q)<\alpha<\omega_{1}^{z, a}\right)\right]$,

where $a \in \mathbb{N}^{\mathbb{N}}$ is an appropriate fixed parameter, independent of $z$. So

$$
\begin{aligned}
& \forall z \exists \alpha<\omega_{1}^{z, a} \exists y \in[h(z)]_{E_{\infty}} \exists n, k, m, p \\
& \quad\left[y \in N_{p} \& \forall q\left(N_{q} \subseteq N_{p} \Rightarrow\left(y \in N_{q} \Leftrightarrow \varphi(f(z), n, k, m, q) \geq \alpha\right)\right)\right] .
\end{aligned}
$$

By boundedness there is some fixed $\alpha_{0}<\omega_{1}$ so that

$$
\begin{aligned}
& \forall z \exists n \exists y \in[h(z)]_{E_{\infty}} \exists \alpha<\alpha_{0} \exists k, m, p \\
& \quad\left[y \in N_{p} \& \forall q\left(N_{q} \subseteq N_{p} \Rightarrow\left(y \in N_{q} \Leftrightarrow \varphi(f(z), n, k, m, q) \geq \alpha\right)\right)\right] .
\end{aligned}
$$

Let, for $\alpha<\alpha_{0}, n, m, p \in \mathbb{N}$,

$$
Z_{n, \alpha, m, p}=\left\{z: \exists y \in[h(z)]_{E_{\infty}} \exists k\left[y \in N_{p} \& \forall q\left(N_{q} \subseteq N_{p} \Rightarrow(*)\right)\right]\right\} .
$$

Then $Z_{n, \alpha, m, p}$ is Borel and $E$-invariant, and $Z=\bigcup_{n, \alpha, m, p} Z_{n, \alpha, m, p}$, so, in the notation of the special case, it is enough to show that $E \mid Z_{n, \alpha, m, p} \leq_{\mathrm{B}}$ $E_{G^{n}}^{Y_{n}}$. For that define $Q_{n, \alpha, m, p}: Z_{n, \alpha, m, p} \rightarrow Y_{n}$ by $Q(z)\left(U_{k}^{n}\right)=$ the unique $y \in[h(z)]_{E_{\infty}}$ such that $y \in N_{p} \& \forall q\left(N_{q} \subseteq N_{p} \Rightarrow(*)\right)$, if such exists; $\infty$ otherwise. This works as in the special case.

8. The Seventh Dichotomy Theorem. Finally, we prove the following result, labeled the Seventh Dichotomy Theorem in [12].

TheOREM 8.1. Let $G \subseteq S_{\infty}$ be a closed subgroup of $S_{\infty}$, admitting an invariant metric. If $X$ is a Borel $G$-space and $E_{G}^{X}$ is Borel, then for any 
$E \leq_{\mathrm{B}} E_{G}^{X}$,

$$
E \leq_{\mathrm{B}} E_{\infty} \quad \text { or } \quad E_{3} \leq_{\mathrm{B}} E .
$$

Proof. We start with the following:

Lemma 1. If $G \subseteq S_{\infty}$ is a closed subgroup of $S_{\infty}$ admitting an invariant metric, there is a sequence $G_{n}$ of countable (discrete) groups so that $G$ is isomorphic to a closed subgroup of $\prod_{n} G_{n}$.

Proof. Fix a conjugation invariant nbhd basis $\left\{V_{n}\right\}$ at the identity. Let $U_{n}$ be the subgroup generated by $V_{n}$. Since $G$ has a nbhd basis at the identity consisting of open subgroups, it follows that $\left\{U_{n}\right\}$ is a nbhd basis consisting of normal open subgroups. Fix an invariant metric $d$ for $G$ and assume without loss of generality that $d\left(U_{n}\right)<2^{-n}$.

Put $\Omega_{n}=G / U_{n}$. Clearly, $\Omega_{n}$ is countable. $G$ acts on $\Omega_{n}$ by $g \cdot h U_{n}=$ $g h U_{n}$. It is easily seen that $g \cdot U_{n}=g^{\prime} \cdot U_{n}$ implies $g \cdot h U_{n}=g h U_{n}=g U_{n} h=$ $g^{\prime} U_{n} h=g^{\prime} h U_{n}=g^{\prime} \cdot h U_{n}$, so $\pi_{g}^{n}\left(h U_{n}\right)=g \cdot h U_{n}$ is completely determined by $\pi_{g}^{n}\left(U_{n}\right)$ and so $\pi_{n}(g)=\pi_{g}^{n}$ is a homomorphism of $G$ onto a countable subgroup $G_{n}$ of the symmetric group of $\Omega_{n}$. If we define $\pi: G \rightarrow \prod_{n} G_{n}$ by $\pi(g)_{n}=\pi_{n}(g)$, clearly $\pi$ is a continuous injection of $G$ into $\prod_{n} G_{n}$. It remains to show that it has continuous inverse on $\pi(G)$. So assume $\pi\left(g_{n}\right) \rightarrow$ $\pi(g)$ in the product topology of $\prod_{n} G_{n}$. Then for any fixed $k$, for all large enough $n$, we have $\pi\left(g_{n}\right)_{k}=\pi(g)_{k}$, i.e., $\pi_{k}\left(g_{n}\right)=\pi_{k}(g)$, so $g_{n} U_{k}=g U_{k}$ and then $g^{-1} g_{n} \in U_{k}$. So $d\left(g^{-1} g_{n}, 1\right)=d\left(g_{n}, g\right)<2^{-k}$, thus $g_{n} \rightarrow g$.

By $[3,2.3 .5]$, if $G$ is a closed subgroup of $H$, then for any Borel $G$-space $X$ there is a Borel $H$-space $Y$ with $E_{G}^{X} \sim_{\mathrm{B}} E_{H}^{Y}$. So it is enough to prove the theorem for $G$ a countable product of countable (discrete) groups and since every such group is a homomorphic image of $\mathbb{F}_{\aleph_{0}}$ (the free group on $\aleph_{0}$ generators) which in turn is a homomorphic image of $H=\bigoplus_{n} \mathbb{F}_{\aleph_{0}}=$ the direct sum of countably many copies of $\mathbb{F}_{\aleph_{0}}$, it is enough to prove it for $G=H^{\mathbb{N}}$. Note that $H^{n+1} \cong H$ for each $n \in \mathbb{N}$.

We will next describe a countable structure with automorphism group $G$. Let

$$
\mathcal{A}_{0}=\left\langle\bigcup_{n=0}^{\infty} H^{n+1},\left\{Q_{n}^{\mathcal{A}_{0}}\right\}_{n \in \mathbb{N}},\left\{F_{h}^{\mathcal{A}_{0}}\right\}_{h \in H},\left\{p_{i j}^{\mathcal{A}_{0}}\right\}_{0 \leq i<j}\right\rangle
$$

be defined as follows: Putting $A_{0}=\bigcup_{n=0}^{\infty} H^{n+1}, Q_{n}$ are unary relations such that

$$
Q_{n}^{\mathcal{A}_{0}}(a) \Leftrightarrow a \in H^{n+1} .
$$

Next, using $H^{n+1} \cong H$ for each $n$, we fix an isomorphism $\varrho_{n}: H \rightarrow H^{n+1}$, say $\varrho_{n}(h)=\left(h_{0}^{n}, \ldots, h_{n}^{n}\right)$. Then each $F_{h}^{\mathcal{A}_{0}}$ is the unary function such that

$$
F_{h}^{\mathcal{A}_{0}}\left(\left(g_{0}, \ldots, g_{n}\right)\right)=\left(g_{0}\left(h_{0}^{n}\right)^{-1}, \ldots, g_{n}\left(h_{n}^{n}\right)^{-1}\right) \text {. }
$$


Thus

$$
F_{h_{1}}^{\mathcal{A}_{0}} \circ F_{h_{2}}^{\mathcal{A}_{0}}=F_{h_{1} h_{2}}^{\mathcal{A}_{0}}
$$

and $F_{h}^{\mathcal{A}_{0}}$ is a permutation of each $\left(Q_{n}\right)^{\mathcal{A}_{0}}$, and in fact $h \cdot\left(g_{0}, \ldots, g_{n}\right)=$ $F_{h}^{\mathcal{A}_{0}}\left(\left(g_{0}, \ldots, g_{n}\right)\right)$ is a free transitive action of $H$ on $\left(Q_{n}\right)^{\mathcal{A}_{0}}$.

Finally, $p_{i j}^{\mathcal{A}_{0}}$ is the unary function defined by

$$
p_{i j}^{\mathcal{A}_{0}}\left(\left(g_{0}, \ldots, g_{n}\right)\right)= \begin{cases}\left(g_{0}, \ldots, g_{n}\right) & \text { if } j \neq n \\ \left(g_{0}, \ldots, g_{i}\right) & \text { if } j=n\end{cases}
$$

We deduce, using $F_{h}^{\mathcal{A}_{0}}, p_{i j}^{\mathcal{A}_{0}}$, that every $a \in\left(Q_{n}\right)^{\mathcal{A}_{0}}$ is definable (by a term) from any $b \in\left(Q_{k}\right)^{\mathcal{A}_{0}}$, if $k \geq n$.

It is clear that every $g=\left(h_{0}, h_{1}, \ldots\right) \in H^{\mathbb{N}}$ gives rise to an automorphism $\varrho_{g}$ of $\mathcal{A}_{0}$ by

$$
\varrho_{g}\left(g_{0}, \ldots, g_{n}\right)=\left(h_{0} g_{0}, \ldots, h_{n} g_{n}\right)
$$

and it is easy to check that every automorphism $\pi$ of $\mathcal{A}_{0}$ is of the form $\varrho_{g}$. Thus $g \mapsto \varrho_{g}$ is an isomorphism of $G$ with $\operatorname{Aut}\left(\mathcal{A}_{0}\right)$.

By a simple coding we can assume that the universe of $\mathcal{A}_{0}$ is $A_{0}=\mathbb{N}$. By identifying $g$ with $\varrho_{g}$ we identify $G=H^{\mathbb{N}}$ with $\operatorname{Aut}\left(\mathcal{A}_{0}\right)$. Denote by $L_{0}$ the language of $\mathcal{A}_{0}$.

Suppose now $X$ is a Borel $G$-space with $E_{G}^{X}$ Borel and $E \leq_{\mathrm{B}} E_{G}^{X}$. As in the proof of Theorem 7.2 we can assume that the Borel reduction is actually $1-1$. By [3, pp. 31-32], the $G$-space $X$ is Borel embeddable in the relativized logic action $J_{L_{0} \cup L}^{\mathcal{A}_{0}}$ (where $L$ is a countable relational language disjoint from $\left.L_{0}\right)$ of $\operatorname{Aut}\left(\mathcal{A}_{0}\right)=G$ on $Y_{L_{0} \cup L}^{\mathcal{A}_{0}}=\left\{\mathcal{M} \in X_{L_{0} \cup L}: \mathcal{M} \mid L_{0}=\mathcal{A}_{0}\right\}$, with $X_{L_{0} \cup L}$ denoting the Polish space of all $L_{0} \cup L$-structures with universe $\mathbb{N}$. The equivalence relation associated with $J_{L_{0} \cup L}^{\mathcal{A}_{0}}$ is $\cong \mid Y_{L_{0} \cup L}^{\mathcal{A}_{0}}$. Let $Y \subseteq Y_{L_{0} \cup L}^{\mathcal{A}_{0}}$ be the range of this $G$-embedding, so $Y$ is an $\cong \mid Y_{L_{0} \cup L}^{\mathcal{A}_{0}}$-invariant subset of $Y_{L_{0} \cup L}^{\mathcal{A}_{0}}$. Clearly, $E_{G}^{X}$ is Borel isomorphic to $\cong Y$. Put

$$
Z=\left\{\mathcal{M} \in X_{L_{0} \cup L}: \exists \mathcal{B} \in Y(\mathcal{B} \cong \mathcal{M})\right\} .
$$

We claim that $Z$ is Borel. This is because

$$
\mathcal{M} \in Z \Leftrightarrow \mathcal{M} \mid L_{0} \cong \mathcal{A}_{0} \& \forall g \in S_{\infty}\left(g \cdot \mathcal{M} \mid L_{0}=\mathcal{A}_{0} \Rightarrow g \cdot \mathcal{M} \in Y\right) .
$$

We now claim that $\cong \mid Z$ is also Borel. This is because for $\mathcal{M}, \mathcal{N} \in Z$, $\mathcal{M} \cong \mathcal{N}$

$\Leftrightarrow \forall g \in S_{\infty} \forall h \in S_{\infty}\left(g \cdot \mathcal{M}\left|L_{0}=\mathcal{A}_{0} \& g \cdot \mathcal{N}\right| L_{0}=\mathcal{A}_{0} \Rightarrow g \cdot \mathcal{M} \cong g \cdot \mathcal{N}\right)$. Of course, there is a sentence $\sigma \in\left(L_{0} \cup L\right)_{\omega_{1}}$ such that $Z=\operatorname{Mod}(\sigma)$.

In summary: We have the countable structure $\mathcal{A}_{0}$ with universe $\mathbb{N}$ in the language $L_{0}$ such that:

(i) $L_{0}=\left\{Q_{n}: n \in \mathbb{N}\right\} \cup\left\{F_{g}: g \in H\right\} \cup\left\{p_{i j}: 0 \leq i<j\right\}$. 
(ii) $\mathbb{N}$ is the disjoint union of $\left(Q_{n}\right)^{\mathcal{A}_{0}}$.

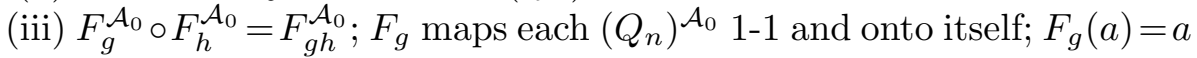
$\Leftrightarrow g=1$, for any $a \in \mathbb{N}, g \in H$.

(iv) If $a \in\left(Q_{n}\right)^{\mathcal{A}_{0}}$, then $p_{i, n}^{\mathcal{A}_{0}}(a) \in\left(Q_{i}\right)^{\mathcal{A}_{0}}$.

Moreover, we have a sentence $\sigma \in\left(L_{0} \cup L\right)_{\omega_{1}}$ such that $\cong \mid \operatorname{Mod}(\sigma)$ is Borel, and every $\mathcal{M} \in \operatorname{Mod}(\sigma)$ is isomorphic to an expansion of $\mathcal{A}_{0}$. Finally, there is a Borel injection $f: W \rightarrow \operatorname{Mod}(\sigma)$, where $E$ lives on $W$, such that

$$
x E y \Leftrightarrow f(x) \cong f(y) \text { and } f[W]=X_{0} \subseteq\left\{\mathcal{A} \in \operatorname{Mod}(\sigma): \mathcal{A} \mid L_{0}=\mathcal{A}_{0}\right\} .
$$

By relativization, we can assume that $L_{0} \cup L$ is recursive, $\sigma \in L_{\omega_{1}^{c k}}$, $\cong \mid \operatorname{Mod}(\sigma)$ is $\Delta_{1}^{1}$, also $f, X_{0} \in \Delta_{1}^{1}$, and $\operatorname{Aut}\left(\mathcal{A}_{0}\right)$ has a dense subgroup consisting of recursive elements. (Notice that $\mathcal{A}_{0}, L_{0}$ are also recursive.)

Fix now for each $n$ an element $p_{n} \in\left(Q_{n}\right)^{\mathcal{A}_{0}}$. Using this we can define an action of $H$ on $\left(Q_{n}\right)^{\mathcal{A}_{0}}$ by

$$
h \cdot F_{h_{1}}\left(p_{n}\right)=F_{h_{1} h^{-1}}\left(p_{n}\right) .
$$

(Notice that every $a \in\left(Q_{n}\right)^{\mathcal{A}_{0}}$ is of the form $F_{h_{1}}\left(p_{n}\right)$ for a unique $h_{1} \in H$.) This defines a homomorphism $\pi: G\left(=H^{\mathbb{N}}\right) \rightarrow S_{\infty}$ by letting $\pi\left(\left(h_{0}, h_{1}, \ldots\right)\right)$ act on $\left(Q_{n}\right)^{\mathcal{A}_{0}}$ via $h_{n}$. It is now easy to check that $\pi(G) \supseteq \operatorname{Aut}\left(\mathcal{A}_{0}\right)$. We put $\pi_{g}=\pi(g)$. By relativization, we can also assume that the closed set $\{g \in G$ : $\left.\pi_{g} \in \operatorname{Aut}\left(\mathcal{A}_{0}\right)\right\}$ admits a countable dense set consisting of recursive elements.

Below, fragment of a language $\mathcal{L}_{\omega_{1} \omega}$ means countable fragment. (The definitions concerning the theory of $\mathcal{L}_{\omega_{1} \omega}$ are as in [2].) For $\mathcal{M}=\langle M,-\rangle$ an $\mathcal{L}$-structure, $F$ a fragment and $\bar{a} \in M^{<\mathbb{N}}$, let $\operatorname{Th}_{F}(M, \bar{a})=\{\varphi \in F$ : $\mathcal{M} \models \varphi(\bar{a})\}$.

If $A \subseteq \operatorname{Mod}(\sigma), \mathcal{M} \in \operatorname{Mod}(\sigma), \bar{a} \in M^{<\mathbb{N}}$ then put

$$
(\mathcal{M}, \bar{a})=A \Leftrightarrow \exists g \in S_{\infty}\left(g\left(a_{i}\right)=a_{i} \text { and } g \cdot \mathcal{M} \in A\right) .
$$

Definition. Let $F \subseteq\left(L_{0} \cup L\right)_{\omega_{1} \omega}$ be a fragment, $A \subseteq \operatorname{Mod}(\sigma), \mathcal{M} \in$ $\operatorname{Mod}(\sigma)$ and $a \in\left(Q_{n}\right)^{\mathcal{M}}, b^{0} \in\left(Q_{k_{0}}\right)^{\mathcal{M}}, \ldots, b^{i} \in\left(Q_{k_{i}}\right)^{\mathcal{M}}$ with $n<k_{0}<$ $\ldots<k_{i}$. We say that $A$ isolates $\operatorname{Th}_{F}(\mathcal{M}, a, \bar{b})$ over $(\mathcal{M}, a)$ if

(i) $(\mathcal{M}, a, \bar{b}) \models A$,

(ii) for all $\mathcal{M}_{0} \in \operatorname{Mod}(\sigma)$ and all $g \in S_{\infty}$ with $g(a)=a$, we have $\left(\mathcal{M}_{0}, a, \bar{b}\right)=A \&\left(g \cdot \mathcal{M}_{0}, a, \bar{b}\right) \models A \Rightarrow \operatorname{Th}_{F}\left(\mathcal{M}_{0}, a, \bar{b}\right)=\operatorname{Th}_{F}\left(g \cdot \mathcal{M}_{0}, a, \bar{b}\right)$.

If $\psi \in\left(L_{0} \cup L\right)_{\omega_{1} \omega}$, we say that $\psi$ isolates $\operatorname{Th}_{F}(\mathcal{M}, a, \bar{b})$ over $(\mathcal{M}, a)$ if

(i) $(\mathcal{M}, a, \bar{b}) \models \psi$ (i.e., $\mathcal{M} \models \psi(a, \bar{b})$ ),

(ii) for all $\mathcal{M}_{0} \in \operatorname{Mod}(\sigma)$, all $a_{0} \in\left(Q_{n}\right)^{\mathcal{M}_{0}}$ and all $b_{0}^{i},\left(b_{0}^{i}\right)^{\prime} \in\left(Q_{k_{i}}\right)^{\mathcal{M}_{0}}$, we have

$$
\begin{aligned}
\left(\mathcal{M}_{0}, a_{0}, \bar{b}_{0}\right) \models \psi \&\left(\mathcal{M}_{0}, a_{0}, \bar{b}_{0}^{\prime}\right) & \models \psi \\
\Rightarrow & \operatorname{Th}_{F}\left(\mathcal{M}_{0}, a_{0}, \bar{b}_{0}\right)=\operatorname{Th}_{F}\left(\mathcal{M}_{0}, a_{0}, b_{0}^{\prime}\right) .
\end{aligned}
$$


Let us note that if $A \subseteq \operatorname{Mod}(\sigma)$ is $\boldsymbol{\Delta}_{1}^{1}$ and invariant under all $g \in S_{\infty}$ with $g(a)=a, g\left(b^{i}\right)=b^{i}$, then by [21], there is a formula $\psi \in\left(L_{0} \cup L\right)_{\omega_{1} \omega}$ with

$$
(\mathcal{N}, a, \bar{b}) \models \psi \Leftrightarrow(\mathcal{N}, a, \bar{b}) \models A \Leftrightarrow \mathcal{N} \in A,
$$

for all $\mathcal{N} \in \operatorname{Mod}(\sigma)$. We check then that if $A$ isolates $\operatorname{Th}_{F}(\mathcal{M}, a, \bar{b})$ over $(\mathcal{M}, a)$, so does $\psi$.

Indeed, assume $\mathcal{M}_{0}, a_{0}, \bar{b}_{0}, \bar{b}_{0}^{\prime}$ are as in (ii) of the definition of isolation for $\psi$ and assume $\left(\mathcal{M}_{0}, a_{0}, \bar{b}_{0}\right) \models \psi,\left(\mathcal{M}_{0}, a_{0}, \bar{b}_{0}^{\prime}\right)=\psi$. Choosing $\widetilde{g} \in S_{\infty}$ with $\widetilde{g}\left(a_{0}\right)=a, \widetilde{g}\left(b_{0}^{i}\right)=b^{i}$, we see that by replacing $\mathcal{M}_{0}$ by $\widetilde{g} \cdot \mathcal{M}_{0}, a_{0}$ by $\widetilde{g}\left(a_{0}\right)=a$, $b_{0}^{i}$ by $\widetilde{g}\left(b_{0}^{i}\right)=b^{i}$, it is enough to assume that $a_{0}=a, \bar{b}_{0}=\bar{b}$. Let then $g \in S_{\infty}$ be such that $g(a)=a, g\left(\left(b_{0}^{i}\right)^{\prime}\right)=b^{i}$. Then we have $\left(\mathcal{M}_{0}, a, \bar{b}\right) \models \psi$, so $\left(\mathcal{M}_{0}, a, \bar{b}\right) \models A$ and $\left(g \cdot \mathcal{M}_{0}, a, \bar{b}\right) \models \psi$, so $\left(g \cdot \mathcal{M}_{0}, a, \bar{b}\right)$ $\models A$, thus, since $A$ isolates $\operatorname{Th}_{F}(\mathcal{M}, a, \bar{b})$ over $(\mathcal{M}, a)$ we get $\operatorname{Th}_{F}\left(\mathcal{M}_{0}, a_{0}, \bar{b}_{0}\right)$ $=\operatorname{Th}_{F}\left(g \cdot \mathcal{M}_{0}, a, \bar{b}\right)=\operatorname{Th}_{F}\left(\mathcal{M}_{0}, a, \bar{b}_{0}^{\prime}\right)$, so we are done.

LEMma 2. If $F, F^{\prime}$ are fragments, $a \in\left(Q_{n}\right)^{\mathcal{M}}$ and for all $k>n$ and all $b \in\left(Q_{k}\right)^{\mathcal{M}}$ there is $\psi \in F^{\prime}$ isolating $\operatorname{Th}_{F}(\mathcal{M}, a, b)$ over $(\mathcal{M}, a)$, then for any $\bar{b}$ as in the preceding definition there is $\psi \in F^{\prime}$ isolating $\operatorname{Th}_{F}(\mathcal{M}, a, \bar{b})$ over $(\mathcal{M}, a)$.

Proof. Fix $\bar{b}=\left(b^{0}, \ldots, b^{i}\right)$ and put $b=b^{i}$. Fix terms $t_{0}, \ldots, t_{i-1}$ with $t_{j}^{\mathcal{M}}(b)=b^{j}$. Let $\psi \in F^{\prime}$ isolate $\operatorname{Th}_{F}(\mathcal{M}, a, b)$ over $(\mathcal{M}, a)$. Put

$$
\psi^{\prime}\left(x, y^{0}, \ldots, y^{i}\right) \Leftrightarrow \psi\left(x, y^{i}\right) \& \bigwedge_{j<i}\left(t_{j}\left(y^{i}\right)=y^{j}\right) .
$$

Then $(\mathcal{M}, a, b) \models \psi^{\prime}$. We claim that $\psi^{\prime}$ isolates $\operatorname{Th}_{F}(\mathcal{M}, a, \bar{b})$ over $(\mathcal{M}, a)$. To check this fix $\mathcal{M}_{0}, a_{0}, \bar{b}_{0}, \bar{b}_{0}^{\prime}$ so that

$$
\left(\mathcal{M}_{0}, a_{0}, \bar{b}_{0}\right) \models \psi^{\prime}, \quad\left(\mathcal{M}_{0}, a_{0}, \bar{b}_{0}^{\prime}\right) \models \psi^{\prime} .
$$

Thus $b_{0}^{j}=t_{j}^{\mathcal{M}_{0}}\left(b_{0}^{i}\right),\left(b_{0}^{\prime}\right)^{j}=t_{j}^{\mathcal{M}_{0}}\left(\left(b_{0}^{\prime}\right)^{i}\right)$ and $\left(\mathcal{M}_{0}, a_{0}, b_{0}^{i}\right) \models \psi,\left(\mathcal{M}_{0}, a_{0},\left(b_{0}^{\prime}\right)^{i}\right)$ $\models \psi$, so $\operatorname{Th}_{F}\left(\mathcal{M}_{0}, a_{0}, b_{0}^{i}\right)=\operatorname{Th}_{F}\left(\mathcal{M}_{0}, a_{0},\left(b_{0}^{\prime}\right)^{i}\right)$. If now $\theta \in \operatorname{Th}_{F}\left(\mathcal{M}_{0}, a_{0}, \bar{b}_{0}\right)$, then $\theta\left(x, t_{0}(y), \ldots, t_{i-1}(y), y\right) \in \operatorname{Th}_{F}\left(\mathcal{M}_{0}, a_{0}, b_{0}^{i}\right)$, so

$$
\theta\left(x, t_{0}(y), \ldots, t_{i-1}(y), y\right) \in \operatorname{Th}_{F}\left(\mathcal{M}_{0}, a_{0},\left(b_{0}^{\prime}\right)^{i}\right),
$$

thus $\theta \in \operatorname{Th}_{F}\left(\mathcal{M}_{0}, a_{0}, \bar{b}_{0}^{\prime}\right)$ and so $\operatorname{Th}_{F}\left(\mathcal{M}_{0}, a_{0}, \bar{b}_{0}\right)=\operatorname{Th}_{F}\left(\mathcal{M}_{0}, a_{0}, \bar{b}_{0}^{\prime}\right)$.

LEMma 3. If $F, F^{\prime}$ are fragments $a \in\left(Q_{n}\right)^{\mathcal{M}}$ and for all $k>n$ and all $b \in\left(Q_{k}\right)^{\mathcal{M}}$ there is $\psi \in F^{\prime}$ isolating $\operatorname{Th}_{F}(\mathcal{M}, a, b)$ over $(\mathcal{M}, a)$, then for any $n^{\prime}>n, a^{\prime} \in\left(Q_{n^{\prime}}\right)^{\mathcal{M}}$ and every $k^{\prime}>n^{\prime}, b \in\left(Q_{k^{\prime}}\right)^{\mathcal{M}}$ there is $\psi \in F^{\prime}$ isolating $\operatorname{Th}_{F}\left(\mathcal{M}, a^{\prime}, b\right)$ over $\left(\mathcal{M}, a^{\prime}\right)$.

Proof. Fix such $n^{\prime}, a^{\prime}, b$. By the preceding lemma there is a formula $\psi \in$ $F^{\prime}$ isolating $\operatorname{Th}_{F}\left(\mathcal{M}, a, a^{\prime}, b\right)$ over $(\mathcal{M}, a)$. Let $t$ be a term with $t^{\mathcal{M}}\left(a^{\prime}\right)=a$. Put

$$
\psi^{\prime}(x, y) \Leftrightarrow \psi(t(x), x, y)
$$


Then $\left(\mathcal{M}, a^{\prime}, b\right) \models \psi$ and we claim that $\psi^{\prime}$ isolates $\left(\mathcal{M}, a^{\prime}, b\right)$ over $\left(\mathcal{M}, a^{\prime}\right)$. Indeed, let $\left(\mathcal{M}_{0}, a_{0}^{\prime}, b_{0}\right)=\psi^{\prime},\left(\mathcal{M}_{0}, a_{0}^{\prime}, b_{0}^{\prime}\right) \models \psi^{\prime}$. If $a_{0}=t^{\mathcal{M}_{0}}\left(a_{0}^{\prime}\right)$, then $\left(\mathcal{M}_{0}, a_{0}, a_{0}^{\prime}, b_{0}\right) \models \psi$ and $\left(\mathcal{M}_{0}, a_{0}, a_{0}^{\prime}, b_{0}^{\prime}\right) \models \psi$, so $\operatorname{Th}_{F}\left(\mathcal{M}_{0}, a_{0}, a_{0}^{\prime}, b_{0}\right)=$ $\operatorname{Th}_{F}\left(\mathcal{M}_{0}, a_{0}, a_{0}^{\prime}, b_{0}\right)$, thus $\operatorname{Th}_{F}\left(\mathcal{M}_{0}, a_{0}^{\prime}, b_{0}\right)=\operatorname{Th}_{F}\left(\mathcal{M}_{0}, a_{0}^{\prime}, b_{0}^{\prime}\right)$.

We now consider 2 cases:

CASE I: $\forall$ fragments $F \subseteq\left(L_{0} \cup L\right)_{\omega_{1} \omega}, F \in L_{\omega_{1}^{c k}} \forall \mathcal{M} \in X_{0} \exists n \exists a \in$ $\left(Q_{n}\right)^{\mathcal{M}} \forall k>n \forall b \in\left(Q_{k}\right)^{\mathcal{M}} \exists A \in \Sigma_{1}^{1}\left(A\right.$ isolates $\operatorname{Th}_{F}(\mathcal{M}, a, b)$ over $\left.(\mathcal{M}, a)\right)$. We will then show that $E \leq_{\mathrm{B}} E_{\infty}$.

Lemma 4. Assume $F \in L_{\omega_{1}^{c k}}$ and $A \in \Sigma_{1}^{1}$ isolates $\operatorname{Th}_{F}(\mathcal{M}, a, b)$ over $(\mathcal{M}, a)$. Then there is $\psi \in L_{\omega_{1}^{c k}}$ which isolates $\operatorname{Th}_{F}(\mathcal{M}, a, b)$ over $(\mathcal{M}, a)$.

Proof. By reflection, as property (ii) in the definition of isolation is $\Pi_{1}^{1}$ in the codes for $A$, there is $\operatorname{Mod}(\sigma) \supseteq A_{0}^{*} \supseteq A, A_{0}^{*} \in \Delta_{1}^{1}$ satisfying (ii), and so having the same isolation property. Let $A_{0}=\left\{\mathcal{M}: \exists g \in S_{\infty}\right.$ $\left.\left(g(a)=a \& g(b)=b \& g \cdot \mathcal{M} \in A_{0}^{*}\right)\right\}$. Then $A_{0}^{*} \subseteq A_{0}$ and $A_{0}^{*}$ still satisfies this property (ii). Clearly, $A_{0}^{*} \in \Sigma_{1}^{1}$. We can repeat effectively this process to get $A \subseteq A_{0}^{*} \subseteq A_{0} \subseteq A_{1}^{*} \subseteq A_{1} \subseteq \ldots$ with $A_{n} \in \Delta_{1}^{1}$ uniformly in $n, A_{n}$ isolating $\operatorname{Th}_{F}(\mathcal{M}, a, b)$ over $(\mathcal{M}, a)$, and $A_{n}$ closed under all $g \in S_{\infty}$ fixing $a, b$. Let $A^{*}=\bigcup_{n} A_{n}$. Then $A^{*} \in \Delta_{1}^{1}$ is invariant under all such $g$ and isolates $\operatorname{Th}_{F}(\mathcal{M}, a, b)$ over $(\mathcal{M}, a)$. If $\psi$ is the corresponding formula, then $\psi \in$ $\left(L_{0} \cup L\right)_{\omega_{1} \omega}, \psi \in L_{\omega_{1}^{c k}}$, and $\psi$ isolates $\operatorname{Th}_{F}(\mathcal{M}, a, b)$ over $(\mathcal{M}, a)$.

Thus $\forall F \in L_{\omega_{1}^{c k}} \forall \mathcal{M} \in X_{0} \exists n \exists a \in\left(Q_{n}\right)^{\mathcal{M}} \forall k>n \forall b \in\left(Q_{k}\right)^{\mathcal{M}}$ $\exists \psi \in L_{\omega_{1}^{c k}}\left(\psi\right.$ isolates $\operatorname{Th}_{F}(\mathcal{M}, a, b)$ over $\left.(\mathcal{M}, a)\right)$. By reflection we can then find, for each fragment $F \in L_{\omega_{1}^{c k}}$, a fragment $F^{+} \in L_{\omega_{1}^{c k}}$ so that $F \subseteq F^{+}$and $F \mapsto F^{+}$is $\Delta_{1}\left(L_{\omega_{1}^{c k}}\right)$ such that $\forall \mathcal{M} \in X_{0} \exists n \exists a \in\left(Q_{n}\right)^{\mathcal{M}}$ $\forall k>n \forall b \in\left(Q_{k}\right)^{\mathcal{M}} \exists \psi \in F^{+}\left(\psi\right.$ isolates $\operatorname{Th}_{F}(\mathcal{M}, a, b)$ over $\left.(\mathcal{M}, a)\right)$.

Define then recursively $\left(F_{\alpha}\right)_{\alpha<\omega_{1}^{c k}}$ by

$$
\begin{aligned}
F_{0} & =\text { the fragment generated by } \sigma, \\
F_{<\alpha} & =\left(\bigcup_{\beta<\alpha} F_{\beta}\right), \\
F_{\alpha} & =\left(F_{<\alpha}\right)^{+},
\end{aligned}
$$

so that each $F_{\alpha} \in L_{\omega_{1}^{c k}}$.

For convenience, given $\mathcal{M} \in \operatorname{Mod}(\sigma), a \in\left(Q_{n}\right)^{\mathcal{M}}, \gamma<\omega_{1}^{c k}$, we say that $(\mathcal{M}, a)$ is $\gamma$-good if for all $k>n$ and all $b \in\left(Q_{k}\right)^{\mathcal{M}}$ there is $\psi \in F_{\gamma}$ isolating $\operatorname{Th}_{F<\gamma}(\mathcal{M}, a, b)$ over $(\mathcal{M}, a)$. Thus for any $\gamma<\omega_{1}^{c k}, \mathcal{M} \in X_{0}$, there are $n$ and $a \in\left(Q_{n}\right)^{\mathcal{M}}$ such that $(\mathcal{M}, a)$ is $\gamma$-good, so that by Lemma 3 , this is also true for any $n^{\prime}>n$.

Below, rank means quantifier rank, as in [2]. 
Lemma 5. Fix $\gamma<\omega_{1}^{c k}$. If $\mathcal{M}, \mathcal{M}_{0} \in X_{0}, a \in\left(Q_{n}\right)^{\mathcal{M}}, a_{0} \in\left(Q_{n}\right)^{\mathcal{M}_{0}}$, $(\mathcal{M}, a),\left(\mathcal{M}_{0}, a_{0}\right)$ are $\gamma$-good and $\operatorname{Th}_{F_{\gamma}}(\mathcal{M}, a)=\operatorname{Th}_{F_{\gamma}}\left(\mathcal{M}_{0}, a_{0}\right)$, then $(\mathcal{M}, a)$, $\left(\mathcal{M}_{0}, a_{0}\right)$ satisfy the same formulas of rank $\gamma$.

Proof. By induction on $\gamma$. For $\gamma=0$ this is obvious as $F_{0} \supseteq$ quantifierfree formulas. Let now $\gamma$ be limit and $\varphi$ be of $\operatorname{rank} \gamma$ with $(\mathcal{M}, a) \models \varphi$. Without loss of generality we can assume that $\varphi=\bigvee_{i} \varphi_{i}$ with each $\varphi_{i}$ of rank $\gamma_{i}<\gamma$. Thus $(\mathcal{M}, a) \models \varphi_{i}$ for some $i$. Fix $n^{\prime}>n$ and $b \in\left(Q_{n^{\prime}}\right)^{\mathcal{M}}$ so that $(\mathcal{M}, b)$ is $\gamma_{i}$-good. As $(\mathcal{M}, a)$ is $\gamma$-good there is $\psi \in F_{\gamma}$ isolating $\operatorname{Th}_{F_{\gamma_{i}}}(\mathcal{M}, a, b)$ over $(\mathcal{M}, a)$. Since $\operatorname{Th}_{F_{\gamma}}(\mathcal{M}, a)=\operatorname{Th}_{F_{\gamma}}\left(\mathcal{M}_{0}, a_{0}\right)$, there is $b_{0} \in\left(Q_{n^{\prime}}\right)^{\mathcal{M}}$ with $\left(\mathcal{M}_{0}, a_{0}, b_{0}\right) \models \psi$. We claim that $\left(\mathcal{M}_{0}, b_{0}\right)$ is $\gamma_{i^{-}}$ good and that $\operatorname{Th}_{F_{\gamma_{i}}}(\mathcal{M}, a, b)=\operatorname{Th}_{F_{\gamma_{i}}}\left(\mathcal{M}_{0}, a_{0}, b_{0}\right)$ so that, in particular, $\operatorname{Th}_{F_{\gamma_{i}}}(\mathcal{M}, b)=\operatorname{Th}_{F_{\gamma_{i}}}\left(\mathcal{M}_{0}, b_{0}\right)$. Then by induction hypothesis $(\mathcal{M}, b)$ and $\left(\mathcal{M}_{0}, b_{0}\right)$ satisfy the same formulas of rank $\gamma_{i}$. But there is a term $t$ such that $t^{\mathcal{M}}(b)=a$ and $t^{\mathcal{M}_{0}}\left(b_{0}\right)=a_{0}$, so as $(\mathcal{M}, a) \models \varphi_{i},(\mathcal{M}, b) \models \varphi_{i}(t)$ and so we have $\left(\mathcal{M}_{0}, b_{0}\right) \models \varphi_{i}(t)$, i.e., $\left(\mathcal{M}_{0}, a_{0}\right) \models \varphi_{i}$, and we are done.

First we check that $\operatorname{Th}_{F_{\gamma_{i}}}(\mathcal{M}, a, b)=\operatorname{Th}_{F_{\gamma_{i}}}\left(\mathcal{M}_{0}, a_{0}, b_{0}\right)$. Suppose $(\mathcal{M}, a, b)=\theta, \theta \in F_{\gamma_{i}}$. Then there is $b_{0}^{\prime} \in\left(Q_{n^{\prime}}\right)^{\mathcal{M}_{0}}$ with $\left(\mathcal{M}_{0}, a_{0}, b_{0}^{\prime}\right) \models \theta$ and $\left(\mathcal{M}_{0}, a_{0}, b_{0}^{\prime}\right)=\psi$, so, as then $\operatorname{Th}_{F_{\gamma_{i}}}\left(\mathcal{M}_{0}, a_{0}, b_{0}\right)=\operatorname{Th}_{F_{\gamma_{i}}}\left(\mathcal{M}_{0}, a_{0}, b_{0}^{\prime}\right)$, we have $\left(\mathcal{M}_{0}, a_{0}, b_{0}\right) \models \theta$.

Next we check that $\left(\mathcal{M}_{0}, b_{0}\right)$ is $\gamma_{i}$-good. Fix $k>n^{\prime}, d_{0} \in\left(Q_{k}\right)^{\mathcal{M}_{0}}$. Take any $d \in\left(Q_{k}\right)^{\mathcal{M}}$ and, since $(\mathcal{M}, b)$ is $\gamma_{i}$-good, let $\varrho^{\prime} \in F_{\gamma_{i}}$ isolate $\operatorname{Th}_{F_{<\gamma_{i}}}(\mathcal{M}, b, d)$ over $(\mathcal{M}, b)$. Then there is $d_{0}^{\prime} \in\left(Q_{k}\right)^{\mathcal{M}_{0}}$ with $\left(\mathcal{M}_{0}, b_{0}, d_{0}^{\prime}\right)$ $=\varrho^{\prime}$. So $\varrho^{\prime}$ isolates $\operatorname{Th}_{F_{<\gamma_{i}}}\left(\mathcal{M}_{0}, b_{0}, d_{0}^{\prime}\right)$ over $\left(\mathcal{M}_{0}, b_{0}\right)$. If $\varrho^{\prime}=\varrho^{\prime}(x, y)$ and we fix $h \in H$ with $F_{h}\left(d_{0}\right)=d_{0}^{\prime}$, we see that $\varrho=\varrho^{\prime}\left(x, F_{h}(y)\right)$ isolates $\operatorname{Th}_{F_{<\gamma_{i}}}\left(\mathcal{M}_{0}, b_{0}, d_{0}\right)$ over $\left(\mathcal{M}_{0}, b_{0}\right)$ and $\varrho \in F_{\gamma_{i}}$, so we are done.

Finally, consider the successor case $\gamma=\delta+1$. Without loss of generality, let $\varphi=\exists x \psi(x)$ be of $\operatorname{rank} \delta+1$ and assume that $(\mathcal{M}, a) \models \varphi$. Then fix $n^{\prime}>n$ and $b \in\left(Q_{n^{\prime}}\right)^{\mathcal{M}}$ so that $(\mathcal{M}, a) \models \psi\left(t^{\mathcal{M}}(b)\right)$ for some term $t$, and $(\mathcal{M}, b)$ is $\delta$-good. As before, we can find $b_{0} \in\left(Q_{n^{\prime}}\right)^{\mathcal{M}_{0}}$ which is $\delta$ good and $\operatorname{Th}_{F_{\gamma}}(\mathcal{M}, a, b)=\operatorname{Th}_{F_{\gamma}}\left(\mathcal{M}_{0}, a_{0}, b_{0}\right)$. Then by induction hypothesis $(\mathcal{M}, b),\left(\mathcal{M}_{0}, b_{0}\right)$ satisfy the same formulas of rank $\delta$, so (as also $a=t_{1}^{\mathcal{M}}\left(b_{0}\right)$, $a_{0}=t_{1}^{\mathcal{M}_{0}}\left(b_{0}\right)$ for some term $\left.t_{1}\right)$, we have $\left(\mathcal{M}_{0}, a_{0}\right) \models \psi\left(t^{\mathcal{M}_{0}}\left(b_{0}\right)\right)$, thus $\left(\mathcal{M}, a_{0}\right) \models \varphi$ and we are done.

Since $\cong \mid \operatorname{Mod}(\sigma)$ is $\Delta_{1}^{1}$, it follows from Vaught's work (see, e.g., $[14,16.9]$ ) that there is $\gamma_{0}<\omega_{1}^{c k}$ such that if $\mathcal{M}, \mathcal{M}_{0}$ are in $\operatorname{Mod}(\sigma)$ and satisfy the same formulas of rank $\gamma_{0}$, then $\mathcal{M} \cong \mathcal{M}_{0}$.

By Kreisel Selection, there is a $\Delta_{1}^{1}$ function which assigns to each $\mathcal{M} \in X_{0}$ some $n_{\mathcal{M}} \in \mathbb{N}$ and $a_{\mathcal{M}} \in\left(Q_{n_{\mathcal{M}}}\right)^{\mathcal{M}}$ so that $\left(\mathcal{M}, a_{\mathcal{M}}\right)$ is $\gamma_{0}$-good. Put, for $\mathcal{M} \in X_{0}$,

$$
U(\mathcal{M})=\operatorname{Th}_{F_{\gamma_{0}}}\left(\mathcal{M}, a_{\mathcal{M}}\right)
$$


Then $U$ is a $\Delta_{1}^{1}$ function (from $X_{0}$ into $2^{F_{\gamma_{0}}}$ ) and, by the preceding lemma, if $\mathcal{M}, \mathcal{M}_{0} \in X_{0}$ and $U(\mathcal{M})=U\left(\mathcal{M}_{0}\right)$, then $\mathcal{M} \cong \mathcal{M}_{0}$. Moreover, for each $\mathcal{M} \in X_{0},\left\{U\left(\mathcal{M}_{0}\right): \mathcal{M}_{0} \in X_{0} \& \mathcal{M} \cong \mathcal{M}_{0}\right\}$ is countable, since it is contained in $\left\{\operatorname{Th}_{F_{\gamma_{0}}}(\mathcal{M}, a): a \in \mathcal{M}\right\}$. It follows that $U$ maps each $\cong \mid X_{0}$-class into a countable set and distinct isomorphic classes are mapped to disjoint sets, so $\cong X_{0} \leq_{\mathrm{B}} E_{\infty}$. As $E_{G}^{X}$ is Borel isomorphic to $\cong \mid X_{0}$, this shows that $E \leq_{\mathrm{B}} E_{G}^{X} \leq_{\mathrm{B}} E_{\infty}$, so $E \leq_{\mathrm{B}} E_{\infty}$.

CASE II: There are $F \in L_{\omega_{1}^{c k}}$ and $\mathcal{M} \in X_{0}$ so that for all $n, a \in\left(Q_{n}\right)^{\mathcal{M}}$ there are $k>n$ and $b \in\left(Q_{k}\right)^{\mathcal{M}}$ such that no $A \in \Sigma_{1}^{1}$ isolates $\operatorname{Th}_{F}(\mathcal{M}, a, b)$ over $(\mathcal{M}, a)$. Fix such an $F$ from now.

Let

$$
\begin{aligned}
X_{0}^{\prime}=\{\mathcal{M} \in \operatorname{Mod}(\sigma): & \left.\mathcal{M} \mid L_{0}=\mathcal{A}_{0} \text { and } \exists \mathcal{N} \in X_{0}(\mathcal{M} \cong \mathcal{N})\right\} \\
Y_{0}^{\prime}=\{\mathcal{M} \in \operatorname{Mod}(\sigma): & \mathcal{M} \mid L_{0}=\mathcal{A}_{0} \text { and } \forall n \forall a \in\left(Q_{n}\right)^{\mathcal{M}} \exists k>n \exists b \in\left(Q_{k}\right)^{\mathcal{M}} \\
& \left.\left(\text { no } A \in \Sigma_{1}^{1} \text { isolates } \operatorname{Th}_{F}(\mathcal{M}, a, b) \text { over }(\mathcal{M}, a)\right)\right\} \\
=\{\mathcal{M} \in \operatorname{Mod}(\sigma): & \mathcal{M} \mid L_{0}=\mathcal{A}_{0} \text { and } \forall n \forall a \in\left(Q_{n}\right)^{\mathcal{M}} \exists k>n \exists b \in\left(Q_{k}\right)^{\mathcal{M}} \\
& \left.\left(\text { no } \psi \in L_{\omega_{1}^{c k}} \text { isolates } \operatorname{Th}_{F}(\mathcal{M}, a, b) \text { over }(\mathcal{M}, a)\right)\right\} .
\end{aligned}
$$

Then $X_{0}^{\prime}, Y_{0}^{\prime}$ are invariant under the action of $\operatorname{Aut}\left(\mathcal{A}_{0}\right)$ (i.e., under $\cong \mid Y_{L_{0} \cup L}^{\mathcal{A}_{0}}$ ) and by the Case II assumption

$$
Y_{0}=X_{0}^{\prime} \cap Y_{0}^{\prime} \neq \emptyset
$$

and clearly $Y_{0} \in \Sigma_{1}^{1}$. We will show that

$$
E_{0}^{\mathbb{N}} \leq_{\mathrm{c}}\left(\cong \mid Y_{0}\right) .
$$

Then $E_{0}^{\mathbb{N}} \leq_{\mathrm{c}}\left(\cong \mid X_{0}^{\prime}\right)$. Since clearly $\left(\cong \mid X_{0}^{\prime}\right) \leq_{C \text {-meas }}\left(\cong \mid X_{0}\right) \sqsubseteq_{\mathrm{B}} E$, it follows that $E_{0}^{\mathbb{N}} \leq_{C \text {-meas }} E$. So there is a comeager set $D$ with $E_{0}^{\mathbb{N}} \mid D \leq_{c} E$. We then claim that $E_{0}^{\mathbb{N}} \sqsubseteq_{\mathrm{c}} E_{0}^{\mathbb{N}} \mid D$, which completes the proof. To see this notice that by the Sixth Dichotomy Theorem it is enough to show that $E_{0}^{\mathbb{N}} \mid D \not \not_{\mathrm{B}} E_{\infty}$. Identifying, as usual, $\left(2^{\mathbb{N}}\right)^{\mathbb{N}}$ with $2^{\mathbb{N} \times \mathbb{N}}$ we see that $E_{0}^{\mathbb{N}}=E_{I_{3}}$. If $E_{0}^{\mathbb{N}}\left|D=E_{I_{3}}\right| D \leq_{\mathrm{B}} E_{\infty}$, then, by [17], $I_{3} \in \Sigma_{2}^{0}$, which is a contradiction, as $I_{3}$ is complete $\Pi_{3}^{0}$.

So it remains to prove $(*)$. We keep the notation $\langle m, j\rangle, L(n)$ from the proof of Theorem 7.2. We assume without loss of generality that $\{0, \ldots$, $n-1\} \subseteq \bigcup_{i<n}\left(Q_{i}\right)^{\mathcal{A}_{0}}$, and for any $\mathcal{M} \in \operatorname{Mod}(\sigma)$ we let $\mathcal{M} \mid n$ be the restriction of $\mathcal{M}$ to $n$ (for this we view $\mathcal{M}$ as relational by replacing functions by their graphs). We also fix a recursive free $T_{\emptyset}$ such that

$$
\mathcal{M} \in Y_{0} \Leftrightarrow \exists y \forall n(\mathcal{M}|n, y| n) \in T_{\emptyset} .
$$

Let also, for each $n$,

$$
V_{n}=\left\{g=\left(h_{0}, h_{1}, \ldots\right) \in H^{\mathbb{N}}: \pi_{g} \in \operatorname{Aut}\left(\mathcal{A}_{0}\right) \& h_{0}=\ldots=h_{n}=1\right\} .
$$

We will define the following, by induction on $n \geq 0$ : 
(i) Non-empty $\Sigma_{1}^{1}$ sets $A_{s}, s \in 2^{n+1}$, with $A_{\emptyset}=Y_{0}, A_{s^{\wedge} i} \subseteq A_{s}$.

(ii) $k_{m} \in \mathbb{N}, m \leq L(n)$, chosen so that $0<k_{0}<k_{1}<\ldots$

(iii) We will also have

$$
\begin{aligned}
\mathcal{M} \in A_{0^{n+1}} \Rightarrow \forall r \leq & L(n) \forall a \in\left(Q_{r}\right)^{\mathcal{M}} \exists b \in\left(Q_{k_{r}}\right)^{\mathcal{M}} \\
& \left(\text { no } A \in \Sigma_{1}^{1} \text { isolates } \operatorname{Th}_{F}(\mathcal{M}, a, b) \text { over }(\mathcal{M}, a)\right) .
\end{aligned}
$$

(iv) Each $A_{s}, s \in 2^{n+1}$, will be invariant under $\pi\left(V_{k_{L(n)}}\right)$.

(v) For each $s \in 2^{n+1}$ we will also have $\mu_{s}, y_{s}$ of length $k_{L(n)}+1$ and $p_{s} \in H^{k_{L(n)}+1}$ such that $\left(\mathcal{M} \mid\left(k_{L(n)}+1\right)=\mu_{s}\right) \& \forall \mathcal{M} \in A_{s} \exists g \in H^{\mathbb{N}}, g \supseteq p_{s}$ $\exists y \supseteq y_{s}\left[\left(\pi_{g} \cdot \mathcal{M}, y\right) \in\left[T_{\emptyset}\right]\right]$. Moreover, $s \subseteq t \Rightarrow \mu_{s} \subseteq \mu_{t}, y_{s} \subseteq y_{t}, p_{s} \subseteq p_{t}$.

$(\mathrm{v})^{\prime}$ We can view $(\mathrm{v})$ as a requirement concerning $A_{s}$ relative to $A_{\emptyset}$ $=Y_{0}$. We will also impose a similar requirement relative to each $A_{s_{0}}$, with $s_{0} \in 2^{n_{0}+1}, n_{0}=\left\langle m_{0}, 0\right\rangle$, for all $A_{s}, s \in 2^{n+1}, n>n_{0}, s \supseteq s_{0}$, i.e., we will fix a free $T_{s_{0}}$ for $A_{s_{0}}$ and define $\mu_{s}^{s_{0}}, y_{s}^{s_{0}}, p_{s}^{s_{0}}$ with similar properties, with the stipulation that $\mu_{s}^{s_{0}}=\mu_{s}$ and $p_{s}^{s_{0}}(i)=1$ if $i \leq k_{L\left(n_{0}\right)}$.

(vi) $g_{s} \in H^{\mathbb{N}}$, for each $s \in 2^{n+1}$, with $g_{0^{n+1}}=1, g_{s}$ recursive, and $\pi_{g_{s}} \in \operatorname{Aut}\left(\mathcal{A}_{0}\right)$.

(vii) Links. We will also have, for $s \in 2^{n+1}$,

$$
\pi_{g_{s}} \cdot A_{0^{n+1}}=A_{s} .
$$

(viii) Positive requirements. For $s, t \in 2^{n+1}$, put $g_{s, t}=g_{t} g_{s}^{-1}$. If $\bar{n}<n$, $(\bar{s}, \bar{t}) \subseteq(s, t), \bar{s}, \bar{t} \in 2^{\bar{n}+1}$, then we must have, for $l \leq L(n)$,

$$
[\forall \bar{l} \leq l \forall\langle\bar{l}, i\rangle \in(n+1) \backslash(\bar{n}+1)(s(\langle\bar{l}, i\rangle)=t(\langle\bar{l}, i\rangle))] \Rightarrow g_{s, t} \equiv_{l} g_{\bar{s}, \bar{t}},
$$

where for $g, h \in H^{\mathbb{N}}, l \in \mathbb{N}$ we let

$$
g \equiv_{l} h \Leftrightarrow \forall i \leq l\left(g_{i}=h_{i}\right) .
$$

(ix) Negative requirements. If $s, t \in 2^{n+1}, n=\langle m, j\rangle$, and $s(n) \neq t(n)$, then

$$
\begin{aligned}
\mathcal{M} \in A_{s} \& g \in H^{\mathbb{N}} \& \pi_{g} \in \operatorname{Aut}\left(\mathcal{A}_{0}\right) \& g(m), g\left(k_{m}\right) \in\left\{\widetilde{h}_{0}, \ldots, \widetilde{h}_{n}\right\} & \\
& \Rightarrow \pi_{g} \cdot \mathcal{M} \notin A_{t},
\end{aligned}
$$

where $\left\{\widetilde{h}_{0}, \widetilde{h}_{1}, \ldots\right\}$ is a recursive enumeration of $H$ with $\widetilde{h}_{0}=1$.

Assume all this can be done. For each $x \in 2^{\mathbb{N}}$, let

$$
\mathcal{M}_{x}=\bigcup_{n} \mu_{x \mid n+1} \text {. }
$$

We first claim that $\mathcal{M}_{x} \in Y_{0}$. To see this let $y_{x}=\bigcup_{n} y_{x \mid n+1}$ and $g_{x}=$ $\bigcup_{n} p_{x \mid n+1} \in H^{\mathbb{N}}$. For each $n$, fix $\mathcal{M}^{n} \in A_{x \mid n+1}$. Then $\mathcal{M}^{n} \mid\left(k_{L(n)}+1\right)=$ $\mathcal{M}_{x} \mid\left(k_{L(n)}+1\right)$, so $\mathcal{M}^{n} \rightarrow \mathcal{M}_{x}$ (in the usual topology of $\left.X_{L_{0} \cup L}\right)$. 
Also, fix $g^{n} \in H^{\mathbb{N}}, g^{n} \supseteq p_{x \mid n+1}$ and $y^{n} \supseteq y_{x \mid n+1}$ with $\left(\pi_{g^{n}} \cdot \mathcal{M}^{n}, y^{n}\right) \in\left[T_{\emptyset}\right]$. Notice that $\pi_{g^{n}} \in \operatorname{Aut}\left(\mathcal{A}_{0}\right)$, as $\mathcal{M}^{n}, \pi_{g^{n}} \cdot \mathcal{M}_{n} \in Y_{0}$, so they are both expansions of $\mathcal{A}_{0}$. As $\pi_{g^{n}} \rightarrow \pi_{g_{x}}, \mathcal{M}^{n} \rightarrow \mathcal{M}_{x}, y^{n} \rightarrow y$ we have $\left(\pi_{g_{x}} \cdot \mathcal{M}_{x}, y\right)$ $\in\left[T_{\emptyset}\right]$, so $\pi_{g_{x}} \cdot \mathcal{M}_{x} \in Y_{0}$, and $\pi_{g_{x}} \in \operatorname{Aut}\left(\mathcal{A}_{0}\right)$, so as $Y_{0}$ is invariant under $\operatorname{Aut}\left(\mathcal{A}_{0}\right)$, we have $\mathcal{M}_{x} \in Y_{0}$.

By (v), $x \mapsto \mathcal{M}_{x}$ is clearly continuous. Finally, we check that

$$
x E_{0}^{\mathbb{N}} y \Leftrightarrow \mathcal{M}_{x} \cong \mathcal{M}_{y} .
$$

Indeed, assume $x E_{0}^{\mathbb{N}} y$. By (viii), there is an element $\left(h_{0}, h_{1}, \ldots\right) \in H^{\mathbb{N}}$ such that $g_{x|n+1, y| n+1} \rightarrow\left(h_{0}, h_{1}, \ldots\right)$. Clearly,

$$
\pi_{g_{x|n+1, y| n+1}} \cdot A_{x \mid n+1}=A_{y \mid n+1},
$$

so if $\mathcal{M}^{n} \in A_{x \mid n+1}$, we have

$$
\pi_{g_{x|n+1, y| n+1}} \cdot \mathcal{M}^{n}=\mathcal{N}^{n} \in A_{y \mid n+1} .
$$

Since $\mathcal{M}^{n} \rightarrow \mathcal{M}_{x}, \mathcal{N}^{n} \rightarrow \mathcal{M}_{y}$ it follows that $\pi_{\left(h_{0}, h_{1}, \ldots\right)} \cdot \mathcal{M}_{x}=\mathcal{M}_{y}$, so $\mathcal{M}_{x} \cong \mathcal{M}_{y}$.

Conversely, assume $\neg x E_{0}^{\mathbb{N}} y$. Fix $m$ such that $x(\langle m, j\rangle) \neq y(\langle m, j\rangle)$ for infinitely many $j$. Assume, towards a contradiction, that $\mathcal{M}_{x} \cong \mathcal{M}_{y}$, and let $g=\left(h_{0}, h_{1}, \ldots\right) \in H^{\mathbb{N}}$ be such that $\pi_{g} \cdot \mathcal{M}_{x}=\mathcal{M}_{y}$. Let $n=\langle m, j\rangle$ be large enough so that $h_{m}, h_{k_{m}} \in\left\{\widetilde{h}_{0}, \ldots, \widetilde{h}_{n}\right\}$. Then by (ix), $\pi_{g} \cdot \mathcal{M}_{x \mid n+1} \cap$ $\mathcal{M}_{y \mid n+1}=\emptyset$. Now by $(\mathrm{v})^{\prime}$, exactly as in the argument that $\mathcal{M}_{x} \in Y_{0}$ (and using the fact that each $A_{s}, s \in 2^{n+1}$, is $\pi\left(V_{k_{L(n)}}\right)$-invariant), we conclude that $\mathcal{M}_{x} \in \bigcap_{n} A_{x \mid n+1}, \mathcal{M}_{y} \in \bigcap_{n} A_{y \mid n+1}$, thus $\pi_{g} \cdot \mathcal{M}_{x} \neq \mathcal{M}_{y}$, a contradiction.

\section{CONSTRUCTION}

We start with $A_{\emptyset}=Y_{0}$. Let also $k_{-1}=0, L(-1)=-1$. Assume now the construction of $A_{s}$ has been done up to level $n$, i.e., for $s \in \bigcup_{k \leq n} 2^{n}(n \geq 0)$, $k_{i}$ has been defined for $i \leq L(n-1)$ and consider $2^{n+1}$; put $n=\langle m, j\rangle$. We consider cases as $j=0$ or $j>0$.

(A) $j=0$, i.e., $n=\langle m, 0\rangle$. Thus $L(n)=L(n-1)+1=m$. We first choose $k_{m}=k_{L(n)}>k_{m-1}$ so that there is $\mathcal{M} \in A_{0^{n}}$ such that for $a \in\left(Q_{k_{m-1}}\right)^{\mathcal{M}}$, $b \in\left(Q_{k_{m}}\right)^{\mathcal{M}}$, no $\Sigma_{1}^{1}$ set isolates $\operatorname{Th}_{F}(\mathcal{M}, a, b)$ over $(\mathcal{M}, a)$. (This can be done as $A_{0^{n}} \subseteq Y_{0}$. Note that if this is true for some $a \in\left(Q_{k_{m-1}}\right)^{\mathcal{M}}, b \in\left(Q_{k_{m}}\right)^{\mathcal{M}}$ then it is true for all such $a, b$.)

Note also that for such an $\mathcal{M}$ and any $a \in\left(Q_{m}\right)^{\mathcal{M}}$, there is $b \in\left(Q_{k_{m}}\right)^{\mathcal{M}}$ with no $\Sigma_{1}^{1}$ set isolating $\operatorname{Th}_{F}(\mathcal{M}, a, b)$ over $(\mathcal{M}, a)$. This is because $m \leq$ $k_{m-1}$. Indeed, fixing such an $a \in\left(Q_{m}\right)^{\mathcal{M}}$, let $a^{\prime} \in\left(Q_{k_{m-1}}\right)^{\mathcal{M}}$ and a term $t$ be such that $t^{\mathcal{M}}\left(a^{\prime}\right)=a$. Then let $b \in\left(Q_{k_{m}}\right)^{\mathcal{M}}$ be such that no $\Sigma_{1}^{1}$ set isolates $\operatorname{Th}_{F}\left(\mathcal{M}, a^{\prime}, b\right)$ over $\left(\mathcal{M}, a^{\prime}\right)$ and let $s$ be a term such that $s(b)$ $=a^{\prime}$. If, towards a contradiction, $A \in \Sigma_{1}^{1}$ isolates $\operatorname{Th}_{F}(\mathcal{M}, a, b)$ over $(\mathcal{M}, a)$, then there is a formula $\psi \in L_{\omega_{1}^{c k}}$ isolating $\operatorname{Th}_{F}(\mathcal{M}, a, b)$ over $(\mathcal{M}, a)$ (by 
Lemma 4). Then the formula $\psi(t(x), y) \wedge s(y)=x$ isolates $\operatorname{Th}_{F}\left(\mathcal{M}, a^{\prime}, b\right)$ over $\left(\mathcal{M}, a^{\prime}\right)$, a contradiction.

So

$$
\begin{aligned}
C=\left\{\mathcal{M} \in A_{0^{n}}: \text { for } a\right. & \in\left(Q_{m}\right)^{\mathcal{M}}, b \in\left(Q_{k_{m}}\right)^{\mathcal{M}} \\
& \text { no } \left.A \in \Sigma_{1}^{1} \text { isolates } \operatorname{Th}_{F}(\mathcal{M}, a, b) \text { over }(\mathcal{M}, a)\right\}
\end{aligned}
$$

is $\Sigma_{1}^{1}$ and non-empty. Since $C \subseteq A_{0^{n}}$ it follows that any $\mathcal{M} \in C$ satisfies condition (iii) as well.

We will next find a recursive $h \in H^{\mathbb{N}}$ with $h \in V_{k_{L(n)}}, \pi_{h} \in \operatorname{Aut}\left(\mathcal{A}_{0}\right)$ and a set $\widehat{A}_{0^{n+1}} \subseteq C, \widehat{A}_{0^{n+1}} \neq \emptyset, \widehat{A}_{0^{n+1}} \in \Sigma_{1}^{1}, \widehat{A}_{0^{n+1}}$ invariant under $\pi\left(V_{k_{L(n)}}\right)$, such that $\pi_{h} \cdot \widehat{A}_{0^{n+1}} \subseteq A_{0^{n}}$, and such that if we let $g_{t \wedge 0}=g_{t}, g_{t^{\wedge} 1}=g_{t} h$ for $t \in 2^{n}$ and $\widehat{A}_{t^{\wedge} 0}=\pi_{g_{t}} \cdot \widehat{A}_{0^{n+1}}=\pi_{g_{t} \wedge_{0}} \cdot \widehat{A}_{0^{n+1}}$ and $\widehat{A}_{t^{\wedge} 1}=\pi_{g_{t}} \cdot\left(\pi_{h} \cdot \widehat{A}_{0^{n+1}}\right)=$ $\pi_{g_{t^{\wedge}}} \cdot \widehat{A}_{0^{n+1}}$, then $\widehat{A}_{s}, s \in 2^{n+1}$, satisfy the negative requirements (ix).

Then it is clear that (vi) is satisfied and (ix) will be satisfied even if we shrink each $\widehat{A}_{s}$. It is also clear, as in the proof of Theorem 7.2 , that since $h \in V_{k_{m-1}} \subseteq V_{m}=V_{k_{L(n)}}$ (as $k_{m-1} \geq m$ ), the positive requirements (viii) are satisfied. Notice that we also have (vii) for the $\widehat{A}_{s}$, and (iv) for $\widehat{A}_{s}$ (as $\pi\left(V_{k_{L(n)}}\right) \cdot \widehat{A}_{s}=\pi\left(V_{k_{L(n)}} g_{s}\right) \cdot \widehat{A}_{0^{n+1}}=\pi\left(g_{s} V_{k_{L(n)}}\right) \cdot \widehat{A}_{0^{n+1}}=g_{s} \cdot\left(\pi\left(V_{k_{L(n)}}\right) \cdot\right.$ $\left.\widehat{A}_{0^{n+1}}\right)=g_{s} \cdot \widehat{A}_{0^{n+1}}=\widehat{A}_{s}$ ), and also (iii) for $\widehat{A}_{0^{n+1}}$ (and thus any subset of it), since $\widehat{A}_{0^{n+1}} \subseteq C$.

It remains to shrink $A_{s}, s \in 2^{n+1}$, to $\widehat{A}_{s}$ to achieve also $(\mathrm{v}),(\mathrm{v})^{\prime}$ and make sure that (iv), (vii) are preserved.

To do this, we fix $\mathcal{M}_{s} \in \widehat{A}_{s}$ so that $\pi_{g_{s}} \cdot \mathcal{M}_{0^{n+1}}=\mathcal{M}_{s}$. By applying (v) to $n-1$, for each $s \in 2^{n+1}$ we have $\mu_{s \mid n} \subseteq \mathcal{M}_{s}, y_{s \mid n}, p_{s \mid n} \in H^{k_{L(n-1)}+1}$, so that for some $g \in H^{\mathbb{N}}, g \supseteq p_{s \mid n}, y \supseteq y_{s}$, we get $\left(\pi_{g} \cdot \mathcal{M}_{s}, y\right) \in\left[T_{\emptyset}\right]$. Let then $\mu_{s}=$ $\mathcal{M}_{s}\left|\left(k_{L(n)}+1\right) \supseteq \mu_{s \mid n}, p_{s}=g\right|\left(k_{L(n)}+1\right) \supseteq p_{s \mid n}, y_{s}=y \mid\left(k_{L(n)}+1\right) \supseteq y_{s \mid n}$. Then let $\widehat{\widehat{A}}_{s}=\left\{x \in \widehat{A}_{s}: \mathcal{M} \mid\left(k_{L(n)}+1\right)=\mu_{s} \& \exists g \in H^{\mathbb{N}}, g \supseteq p_{s} \exists y \supseteq y_{s}\right.$ $\left.\left(\left(\pi_{g} \cdot \mathcal{M}, y\right) \in\left[T_{\emptyset}\right]\right)\right\}$. So $\widehat{\widehat{A}}_{s} \in \Sigma_{1}^{1}$ and $\mathcal{M}_{s} \in \widehat{\widehat{A}}_{s} \subseteq \widehat{A}_{s}$.

Put $A_{0^{n+1}}^{\prime}=\bigcap_{s \in 2^{n+1}} \pi_{g_{s}}^{-1} \cdot \widehat{\widehat{A}}_{s}$ and $A_{s}^{\prime}=\pi_{g_{s}} \cdot A_{0^{n+1}}^{\prime}$. Then (v), (vii) are satisfied for $A_{s}^{\prime}$.

We have dealt only with (v) for notational simplicity, but it is clear that fixing witnesses for each $\mathcal{M}_{s}$ with respect to all relevant $A_{s_{0}}, T_{s_{0}}$ we can make sure that actually both $(\mathrm{v}),(\mathrm{v})^{\prime}$ are satisfied by $\widehat{\widehat{A}}_{s}$, and hence $A_{s}^{\prime}$. So it only remains to modify $A_{s}^{\prime}$ to $A_{s} \subseteq \widehat{A}_{s}$ to satisfy (iv) without affecting (v), (v) $)^{\prime}$ (vii). But this is clear if we just take $A_{s}=\pi\left(V_{k_{L(n)}}\right) \cdot A_{s}^{\prime}$ and notice that by arranging that $(\mathrm{v}),(\mathrm{v})^{\prime}$ remain unaffected, since $g \in V_{k_{L(n)}} \Rightarrow$ $\left(\pi_{g} \cdot \mathcal{M}\right)\left(\mid k_{L(n)}+1\right)=\mathcal{M} \mid\left(k_{L(n)}+1\right)$ and $h g\left|\left(k_{L(n)}+1\right)=h\right|\left(k_{L(n)}+1\right)$ for 
any $h \in H^{\mathbb{N}}$. But also

$$
\begin{aligned}
\pi_{g_{s}} \cdot A_{0^{n+1}} & =\pi_{g_{s}} \cdot\left(\pi\left(V_{k_{L(n)}}\right) \cdot A_{0^{n+1}}^{\prime}\right) \\
& =\pi\left(g_{s} V_{k_{L(n)}}\right) \cdot A_{0^{n+1}}^{\prime}=\pi\left(V_{k_{L(n)}} g_{s}\right) \cdot A_{0^{n+1}}^{\prime} \\
& =\pi\left(V_{k_{L(n)}}\right) \cdot\left(\pi_{g_{s}} \cdot A_{0^{n+1}}^{\prime}\right)=\pi\left(V_{k_{L(n)}}\right) \cdot A_{s}^{\prime}=A_{s}
\end{aligned}
$$

so (vii) remains true as well.

So it only remains to find $h, \widehat{A}_{0^{n+1}}$ satisfying the earlier specifications. The key claim is the following:

LEMMA 6. Fix a finite set $S \subseteq H$. There are $\mathcal{M} \in C, b \in\left(Q_{k_{m}}\right)^{\mathcal{M}}$, $h \in V_{m}$ such that for all $g_{1}, g_{2} \in S$,

$$
\mathrm{Th}_{F}\left(\mathcal{M}, F_{g_{1}}\left(p_{m}\right), F_{g_{2}}(b)\right) \neq \mathrm{Th}_{F}\left(\mathcal{M}, p_{m}, p_{k_{m}}\right),
$$

$\pi_{h} \cdot \mathcal{M} \in C$, and $\pi_{h}(b)=p_{k_{m}}$. (Recall that we have previously fixed $p_{n} \in$ $\left(Q_{n}\right)^{\mathcal{A}_{0}}$.)

Let us assume this and proceed to complete the construction. Let

$$
S_{n}=\left\{g_{t_{1}}(m) \widetilde{h}_{i}^{ \pm 1} g_{s_{1}}(m)^{-1}, g_{t_{1}}\left(k_{m}\right) \widetilde{h}_{i}^{ \pm 1} g_{s_{1}}\left(k_{m}\right)^{-1}: t_{1}, s_{1} \in 2^{n}, i \leq n\right\} .
$$

Let $\mathcal{M}, b, h$ come from Lemma 6 for this $S_{n}$. For $g_{1}, g_{2} \in S_{n}$ fix a formula $\psi_{g_{1}, g_{2}}(x, y) \in F$ with $\mathcal{M}=\psi_{g_{1}, g_{2}}\left(p_{m}, p_{k_{m}}\right)$ but $\mathcal{M}=\neg \psi_{g_{1}, g_{2}}\left(F_{g_{1}}\left(p_{m}\right), F_{g_{2}}(b)\right)$. Let

$$
\psi(x, y)=\left(\bigwedge_{g_{1}, g_{2} \in S_{n}} \psi_{g_{1}, g_{2}}(x, y)\right)
$$

and $\theta(x, y)=\bigwedge_{g_{1}, g_{2} \in S_{n}} \neg \psi\left(F_{g_{1}}(x), F_{g_{2}}(y)\right)$. Then we have $\theta(x, y) \quad=$ $\neg \psi\left(F_{g_{1}}(x), F_{g_{2}}(y)\right)$ for all $g_{1}, g_{2} \in S_{n}$ and $\mathcal{M}=\psi\left(p_{m}, p_{k_{m}}\right), \mathcal{M}=\theta\left(p_{m}, b\right)$. Now notice that if $\mathcal{M}, b, h$ satisfy the lemma, so do $\mathcal{M}, b, h^{\prime}$ for any $h^{\prime} \in h V_{k_{m}}$. Since $h V_{k_{m}}$ is an open set in $\left\{g \in H^{\mathbb{N}}: \pi_{g} \in \operatorname{Aut}\left(\mathcal{A}_{0}\right)\right\}$, there is a recursive $h^{\prime} \in h V_{k_{m}}$, so we can assume without loss of generality that $h$ itself is recursive.

Now let

$$
\widehat{A}_{0^{n+1}}=\left\{\mathcal{M} \in C: \pi_{h} \cdot \mathcal{M} \in C \& \mathcal{M}=\psi\left(p_{m}, p_{k_{m}}\right) \wedge \theta\left(p_{m}, b\right)\right\},
$$

and define the corresponding $\widehat{A}_{s}, g_{s}$ for $s \in 2^{n+1}$, as described earlier. All the other required properties are true, so it is enough to verify that they satisfy the negative requirements (ix).

Assume not, towards a contradiction, and fix $s_{1}, t_{1} \in 2^{n}, \mathcal{M}_{0} \in \widehat{A}_{s_{1} \wedge}$, $\pi_{g} \in \operatorname{Aut}\left(\mathcal{A}_{0}\right)$, with $g(m), g\left(k_{m}\right) \in\left\{\widetilde{h}_{0}^{ \pm 1}, \ldots, \widetilde{h}_{n}^{ \pm 1}\right\}$, and $\pi_{g} \cdot \mathcal{M}_{0} \in \widehat{A}_{t_{1} \wedge 1}$. Then there is $\mathcal{M} \in \widehat{A}_{0^{n+1}}$ such that $\pi_{g_{t_{1}}^{-1} g g_{s_{1}}} \cdot \mathcal{M} \in \pi_{h} \cdot \widehat{A}_{0}$, so there is $\mathcal{M}^{\prime} \in \widehat{A}_{0^{n+1}}$ such that $\mathcal{N}=\pi_{g_{t_{1}}^{-1} g g_{s_{1}}} \cdot \mathcal{M}=\pi_{h} \cdot \mathcal{M}^{\prime}$. Let $g_{t_{1}}^{-1} g g_{s_{1}}(m)=$ $g_{1}^{-1}, g_{t_{1}}^{-1} g g_{s_{1}}\left(k_{m}\right)=g_{2}^{-1}$, so that $g_{1}, g_{2} \in S_{n}, \pi_{g_{t_{1}}^{-1} g g_{s_{1}}}\left(p_{m}\right)=F_{g_{1}}\left(p_{m}\right)$, 
$\pi_{g_{t_{1}}^{-1} g g_{s_{1}}}\left(p_{k_{m}}\right)=F_{g_{2}}\left(p_{k_{m}}\right)$. Then, as $\mathcal{M} \models \psi\left(p_{m}, p_{k_{m}}\right)$, we have $\mathcal{N} \models$ $\psi\left(F_{g_{1}}\left(p_{m}\right), F_{g_{2}}\left(p_{k_{m}}\right)\right)$. But also $\mathcal{M}^{\prime}=\theta\left(p_{m}, b\right)$ and $\pi_{h}\left(p_{m}\right)=p_{m}, \pi_{h}(b)=$ $p_{k_{m}}$, so $\mathcal{N} \models \theta\left(p_{m}, p_{k_{m}}\right)$, contradicting $\theta(x, y) \models \neg \psi\left(F_{g_{1}}(x), F_{g_{2}}(y)\right)$.

So it remains to give the

Proof of Lemma 6. Assume it fails, towards a contradiction. Then for any given $\mathcal{M} \in C, b \in\left(Q_{k_{m}}\right)^{\mathcal{M}}, h \in V_{m}$ with $\pi_{h} \cdot \mathcal{M} \in C, \pi_{h}(b)=p_{k_{m}}$, we have

$$
\operatorname{Th}_{F}\left(\mathcal{M}, F_{g_{1}}\left(p_{m}\right), F_{g_{2}}(b)\right)=\operatorname{Th}_{F}\left(\mathcal{M}, p_{m}, p_{k_{m}}\right)
$$

for some $g_{1}, g_{2} \in S$, and thus

$$
\operatorname{Th}_{F}\left(\mathcal{M}, p_{m}, b\right)=\operatorname{Th}_{F}\left(\mathcal{M}, F_{g_{1}^{-1}}\left(p_{m}\right), F_{g_{2}^{-1}}\left(p_{k_{m}}\right)\right),
$$

so for any fixed $\mathcal{M} \in C$, if $B_{\mathcal{M}}=\left\{b \in\left(Q_{k_{m}}\right)^{\mathcal{M}}: \exists h \in V_{m}\left(\pi_{h} \cdot \mathcal{M} \in C\right.\right.$, $\left.\left.\pi_{h}(b)=p_{k_{m}}\right)\right\}$ then $\left\{\operatorname{Th}_{F}\left(\mathcal{M}, p_{m}, b\right): b \in B_{\mathcal{M}}\right\}$ is finite. Enumerating $F=\left\{\varphi_{0}, \varphi_{1}, \ldots\right\}$, we see then that for every $\mathcal{M} \in C$, there must be some $N \in \mathbb{N}$ such that for any $b \in B_{\mathcal{M}}$,

$$
\begin{aligned}
\forall i \leq N\left(\varphi_{i} \in \operatorname{Th}_{F}\left(\mathcal{M}, p_{m}, b\right)\right. & \left.\Leftrightarrow \varphi_{i} \in \operatorname{Th}_{F}\left(\mathcal{M}, p_{m}, p_{k_{m}}\right)\right) \\
& \Rightarrow \operatorname{Th}_{F}\left(\mathcal{M}, p_{m}, b\right)=\operatorname{Th}_{F}\left(\mathcal{M}, p_{m}, p_{k_{m}}\right) .
\end{aligned}
$$

For each $b \in B_{\mathcal{M}}$, we let

$$
\psi_{b}=\bigwedge_{i \leq N}\left\{\varphi_{i}: \mathcal{M}=\varphi_{i}\left(p_{m}, b\right)\right\} \wedge \bigwedge_{i \leq N}\left\{\neg \varphi_{i}: \mathcal{M} \models \neg \varphi_{i}\left(p_{m}, b\right)\right\},
$$

so that $\mathcal{M}=\psi_{b}\left(p_{m}, b\right)$. If then $\left\{\psi_{0}, \ldots, \psi_{k}\right\}=\left\{\psi_{b}: b \in B_{\mathcal{M}}\right\}$, we see that if $b \in B_{\mathcal{M}}$ and $h \in V_{m}$ with $\pi_{h} \cdot \mathcal{M} \in C, \pi_{h}(b)=p_{k_{m}}$, then

$$
\pi_{h} \cdot \mathcal{M}=\psi_{0}\left(p_{m}, p_{k_{m}}\right) \vee \ldots \vee \psi_{k}\left(p_{m}, p_{k_{m}}\right),
$$

together with

$$
\pi_{h} \cdot \mathcal{M}=\psi_{i}\left(p_{m}, p_{k_{m}}\right), \quad \mathcal{M} \models \psi_{i}\left(p_{m}, p_{k_{m}}\right)
$$

for some $i \leq k$, implies that

$$
\operatorname{Th}_{F}\left(\mathcal{M}, p_{m}, b\right)=\operatorname{Th}_{F}\left(\mathcal{M}, p_{m}, p_{k_{m}}\right) .
$$

Thus we see that

$$
\begin{aligned}
\forall \mathcal{M} \in C & \exists\left\{\psi_{0}, \ldots, \psi_{k}\right\} \subseteq F\left[\forall b \in B_{\mathcal{M}} \forall h \in V_{m}\right. \\
& \left(\pi_{h} \cdot \mathcal{M} \in C \& \pi_{h}(b)=p_{k_{m}} \Rightarrow \pi_{h} \cdot \mathcal{M} \models \bigvee_{i \leq k} \psi_{i}\left(p_{m}, p_{k_{m}}\right)\right) \\
& \wedge \forall i \leq k \forall b \in B_{\mathcal{M}} \forall h \in V_{m}\left[\left(\pi_{h} \cdot \mathcal{M} \in C \& \pi_{h}(b)=p_{k_{m}}\right.\right. \\
& \left.\& \pi_{h} \cdot \mathcal{M} \models \psi_{i}\left(p_{m}, p_{k_{m}}\right) \& \mathcal{M}=\psi_{i}\left(p_{m}, p_{k_{m}}\right)\right) \\
& \left.\left.\Rightarrow \operatorname{Th}_{F}\left(\mathcal{M}, p_{m}, b\right)=\operatorname{Th}_{F}\left(\mathcal{M}, p_{m}, p_{k_{m}}\right)\right]\right]
\end{aligned}
$$


so by $\Pi_{1}^{1}$-uniformization on $\mathbb{N}$ we can find $C_{0} \subseteq C, C_{0}$ non-empty $\Sigma_{1}^{1}$ on which these $\left\{\psi_{0}, \ldots, \psi_{k}\right\}$ are constant, say $\left\{\bar{\psi}_{0}, \ldots, \bar{\psi}_{k_{0}}\right\}$. Taking $b=p_{k_{m}}$, $h=1$, we see that for any $\mathcal{M} \in C_{0}$ there is some $i \leq k_{0}$ with $\mathcal{M} \models$ $\bar{\psi}_{i}\left(p_{m}, p_{k_{m}}\right)$, so fix $i_{0} \leq k_{0}$ such that

$$
C_{1}=\left\{\mathcal{M} \in C_{0}: \mathcal{M} \models \bar{\psi}_{i_{0}}\left(p_{m}, p_{k_{m}}\right)\right\}
$$

is non-empty and clearly $\Sigma_{1}^{1}$. If $\mathcal{M} \in C_{1}$ then we claim that $C_{1}$ isolates $\operatorname{Th}_{F}\left(\mathcal{M}, p_{m}, p_{k_{m}}\right)$ over $\left(\mathcal{M}, p_{m}\right)$, violating the fact that $\mathcal{M} \in C$. Indeed, first $\left(\mathcal{M}, p_{m}, p_{k_{m}}\right) \models C_{1}$ as $\mathcal{M} \in C_{1}$. Now fix $\mathcal{M}_{0} \in \operatorname{Mod}(\sigma), g \in S_{\infty}$ with $g\left(p_{m}\right)=p_{m}$, and $\left(\mathcal{M}_{0}, p_{m}, p_{k_{m}}\right) \models C_{1},\left(g \cdot \mathcal{M}_{0}, p_{m}, p_{k_{m}}\right) \models C_{1}$, in order to show that $\operatorname{Th}_{F}\left(\mathcal{M}_{0}, p_{m}, p_{k_{m}}\right)=\operatorname{Th}_{F}\left(g \cdot \mathcal{M}_{0}, p_{m}, p_{k_{m}}\right)$. Then $h_{1} \cdot \mathcal{M}_{0} \in C_{1}$, $h_{2} g \cdot \mathcal{M}_{0}=h_{2} g h_{1}^{-1} \cdot\left(h_{1} \cdot \mathcal{M}_{0}\right) \in C_{1}$ for some $h_{1}, h_{2} \in S_{\infty}$ that fix $p_{m}, p_{k_{m}}$, so it is enough to show that if $\mathcal{M} \in C_{1}, h^{\prime} \cdot \mathcal{M} \in C_{1}, h^{\prime}$ fixes $p_{m}$ then $\operatorname{Th}_{F}\left(\mathcal{M}, p_{m}, p_{k_{m}}\right)=\operatorname{Th}_{F}\left(h^{\prime} \cdot \mathcal{M}, p_{m}, p_{k_{m}}\right)$. As $\mathcal{M}, h^{\prime} \cdot \mathcal{M}$ are expansions of $\mathcal{A}_{0}$, we see that $h^{\prime} \in \operatorname{Aut}\left(\mathcal{A}_{0}\right)$, so $h^{\prime}=\pi_{h}$ for some $h \in H^{\mathbb{N}}$. As $h^{\prime}$ fixes $p_{m}$ and every $a \in\left(Q_{i}\right)^{\mathcal{A}_{0}}$ for $i \leq m$ is definable from $p_{m}, h^{\prime}$ fixes all such $\left(Q_{i}\right)^{\mathcal{A}_{0}}$, so $h \in V_{m}$. Let $b=\left(\pi_{h}\right)^{-1}\left(p_{k_{m}}\right)$. Then we have $\pi_{h} \cdot \mathcal{M}=\bar{\psi}_{i_{0}}\left(p_{m}, p_{k_{m}}\right)$ as $\pi_{h} \cdot \mathcal{M} \in C_{1}$, and $\mathcal{M}=\bar{\psi}_{i_{0}}\left(p_{m}, p_{k_{m}}\right)$ as $\mathcal{M} \in C_{1}$. So $\operatorname{Th}_{F}\left(\pi_{h} \cdot \mathcal{M}, p_{m}, p_{k_{m}}\right)=$ $\operatorname{Th}_{F}\left(\mathcal{M}, p_{m}, b\right)=\operatorname{Th}_{F}\left(\mathcal{M}, p_{m}, p_{k_{m}}\right)$.

(B) $n=\langle m, j\rangle$ with $j>0$. Then $L(n)=L(n-1)$. Thus $k_{r}$ has already been defined for all $r \leq L(n)$ and by (iii) for $A_{0^{n}}$ we have

$$
\begin{aligned}
\mathcal{M} \in A_{0^{n}} \Rightarrow \forall r \leq L(n) \forall a \in\left(Q_{r}\right)^{\mathcal{M}} \exists b \in\left(Q_{k_{r}}\right)^{\mathcal{M}} & \\
& \quad\left(\text { no } A \in \Sigma_{1}^{1} \text { isolates } \operatorname{Th}_{F}(\mathcal{M}, a, b) \text { over }(\mathcal{M}, a)\right) .
\end{aligned}
$$

So the proof in this case proceeds by defining $C$ exactly as before, i.e.,

$$
\begin{aligned}
C=\left\{\mathcal{M} \in A_{0^{n}}: \text { for } a\right. & \in\left(Q_{m}\right)^{\mathcal{M}}, b \in\left(Q_{k_{m}}\right)^{\mathcal{M}} \\
& \text { no } \left.A \in \Sigma_{1}^{1} \text { isolates } \operatorname{Th}_{F}(\mathcal{M}, a, b) \text { over }(\mathcal{M}, a)\right\}
\end{aligned}
$$

and then literally repeating the rest of the proof of Case (A).

\section{References}

[1] S. Adams and A. S. Kechris, Linear algebraic groups and countable Borel equivalence relations, preprint, 1999.

[2] J. Barwise, Admissible Sets and Structures, Springer, 1975.

[3] H. Becker and A. S. Kechris, The Descriptive Set Theory of Polish Group Actions, London Math. Soc. Lecture Note Ser. 232, Cambridge Univ. Press, Cambridge, 1996.

[4] R. Dougherty, S. Jackson and A. S. Kechris, The structure of hyperfinite Borel equivalence relations, Trans. Amer. Math. Soc. 341 (1994), 193-225.

[5] I. Farah, Ideals induced by Tsirelson submeasures, Fund. Math. 159 (1999), 243-258.

[6] -, Basis problem for turbulent actions. I. Tsirelson submeasures, Ann. Pure Appl. Logic 108 (2001), 189-203. 
[7] I. Farah, Basis problem for turbulent actions. II. $c_{0}$-equalities, Proc. London Math. Soc. 82 (2001), 1-30.

[8] L. A. Harrington, A. S. Kechris and A. Louveau, A Glimm-Effros dichotomy for Borel equivalence relations, J. Amer. Math. Soc. 3 (1990), 903-928.

[9] G. Hjorth, Actions by the classical Banach spaces, J. Symbolic Logic 65 (2000), 392-420.

[10] - Classification and Orbit Equivalence Relations, Math. Surveys Monogr. 75, Amer. Math. Soc., 2000.

[11] G. Hjorth and A. S. Kechris, Borel equivalence relations and classifications of countable models, Ann. Pure Appl. Logic 82 (1996), 221-272.

[12] - - - New dichotomies for Borel equivalence relations, Bull. Symbolic Logic 3 (1997), 329-346.

[13] S. Jackson, A. S. Kechris and A. Louveau, Countable Borel equivalence relations, in preparation, 1999.

[14] A. S. Kechris, Classical Descriptive Set Theory, Grad. Texts in Math. 156, Springer, New York, 1995.

[15] —, Lectures on definable group actions and equivalence relations, unpublished manuscript, 1994.

[16] A. S. Kechris and A. Louveau, The classification of hypersmooth Borel equivalence relations, J. Amer. Math. Soc. 10 (1997), 215-242.

[17] A. Louveau, On the reducibility order between Borel equivalence relations, in: Stud. Logic Found. Math. 134, North-Holland, 1994, 151-155.

[18] A. Louveau and B. Veličković, A note on Borel equivalence relations, Proc. Amer. Math. Soc. 120 (1994), 255-259.

[19] J. H. Silver, Counting the number of equivalence classes of Borel and coanalytic equivalence relations, Ann. Math. Logic 18 (1980), 1-28.

[20] S. Solecki, Analytic ideals, Bull. Symbolic Logic 2 (1996), 339-348.

[21] R. Vaught, Invariant sets in topology and logic, Fund. Math. 82 (1974), 269-294.

[22] B. Veličković, A note on Tsirelson type ideals, ibid. 159 (1999), 259-268.

[23] R. Zimmer, Ergodic Theory and Semisimple Groups, Birkhäuser, 1984.

Department of Mathematics

UCLA

Los Angeles, CA 90095-1555, U.S.A.

E-mail: greg@math.ucla.edu
Department of Mathematics Caltech

Pasadena, CA 91125, U.S.A. E-mail: kechris@caltech.edu

Received 4 October 1999;

in revised form 5 September 2000 